\title{
Modular Groups of Quantum Fields in Thermal States
}

\author{
H.J. BORCHERS \\ Institut für Theoretische Physik \\ Universität Göttingen \\ Bunsenstrasse 9, D 37073 Göttingen \\ and \\ J. YNGVASON \\ Institut für Theoretische Physik \\ Universität Wien \\ Boltzmanngasse 5, A 1090 Wien
}

\begin{abstract}
:
For a quantum field in a thermal equilibrium state we discuss the group generated by time translations and the modular action associated with an algebra invariant under half-sided translations. The modular flows associated with the algebras of the forward light cone and a spacelike wedge admit a simple geometric description in two dimensional models that factorize in light-cone coordinates. At large distances from the domain boundary compared to the inverse temperature the flow pattern is essentially the same as time translations, whereas the zero temperature results are approximately reproduced close to the edge of the wedge and the apex of the cone. Associated with each domain there is also a one parameter group with a positive generator, for which the thermal state is a ground state. Formally, this may be regarded as a certain converse of the Unruh-effect.
\end{abstract}

\section{Introduction}

Algebraic quantum field theory in the sense of Araki, Haag, Kastler [Ha96] is concerned with von Neumann algebras $\mathcal{M}(\mathcal{O})$ of observables localized in space time domains $\mathcal{O}$, together with states $\omega$ on these algebras satisfying some physical selection criterion. Due to the Reeh-Schlieder property of quantum field theory [RS61] one may associate with certain regions $\mathcal{O}$ and states $\omega$ the Tomita-Takesaki modular objects $\Delta_{\mathcal{O}, \omega}$ and $J_{\mathcal{O}, \omega}$ [Ta70], [KR86]. The positive operator $\Delta_{\mathcal{O}, \omega}$ generates a one parameter group ad $\Delta_{\mathcal{O}, \omega}^{\mathrm{it}}$ of automorphisms of $\mathcal{M}(\mathcal{O})$, and the conjugation ad $J_{\mathcal{O}, \omega}$ defined by the antiunitary $J_{\mathcal{O}, \omega}$ maps $\mathcal{M}(\mathcal{O})$ onto its commutant in the GNS Hilbert space corresponding to $\omega$.

Important structural properties of the theory are encoded in the modular objects, see, e.g., [BDL90], [Bch95], but an explicit description of $\Delta_{\mathcal{O}, \omega}^{\mathrm{it}}$ and $J_{\mathcal{O}, \omega}$ has so far only been obtained in the following cases with $\omega$ a vacuum state:

(a) $\mathcal{O}$ is a space like wedge and the local algebras are generated by Wightman fields that transform covariantly with a finite dimensional representation of the Lorentz group [BW75,76].

(b) $\mathcal{O}$ is a forward light cone and $\mathcal{M}(\mathcal{O})$ is generated by a massless, non-interacting field [Bu78].

(c) $\mathcal{O}$ is a double cone and $\mathcal{M}(\mathcal{O})$ is generated by conformally covariant fields [HL82]. 
(d) $\mathcal{O}$ is a space like wedge and the local algebras are generated by generalized free fields of a certain type which break Lorentz covariance [Y93].

In case (a) the modular group is the group of Lorentz boosts that leave the wedge invariant, and the conjugation is the PCT operator (combined with a rotation). Cases (b) and (c) can be reduced to case (a) by a conformal mapping onto the wedge of the forward light cone and the double cone respectively. In (b) the modular group is the dilation group, and in (c) it consists of the conformal transformations that leave the double cone invariant. In the examples considered in (d) the action of the modular group is in general non-local, i.e., an algebra $\mathcal{M}\left(\mathcal{O}_{1}\right)$ with $\mathcal{O}_{1}$ a bounded subset of the wedge need not be mapped into an $\mathcal{M}\left(\mathcal{O}_{2}\right)$ with $\mathcal{O}_{2}$ bounded.

A key to a general understanding of possible geometric interpretations of modular groups is the interplay between the modular action and certain subgroups of the spacetime translations. In [Bch92] it was shown that the modular group of a space-like wedge in a vacuum state acts on the translation group like the Lorentz boosts that leave the wedge invariant. Subsequently Wiesbrock [Wie93] introduced the concept of a half-sided modular inclusion and proved a certain converse of the results of [Bch92], namely that the two-dimensional translation group can be recovered from the modular groups of the wedge and some of its translates.

In this paper we want to investigate the modular groups when $\omega$ is a thermodynamic equilibrium state (KMS state) rather than a vacuum state. In the next Section 2 we discuss the generalizations of the results of [Bch92] to KMS states. We investigate the commutation relations between the time translations and the modular group in a KMS state for any domain that is mapped into itself under half-sided time translations. Using the results of [Bch95] we prove that the time translations and modular action together give rise to a representation of the abstract Lie group generated by one dimensional dilations and translations. The important observation that half-sided modular actions always lead to a representation of this group was first made by Wiesbrock [Wie93], [Wie97]. We express all its one parameter subgroups in terms of the translations and the modular group. Of particular interest is a subgroup with a positive generator. This group acts on the global observable algebra for positive values of the group parameter.

The group relations alone do not determine the modular action and the group with positive generator, but they put definite restrictions on the possible disclocalization of observables by the group actions. More precisely, if $\mathcal{N}$ denotes the observable algebra of a domain invariant under half sided translations and $\mathcal{N}(t)$ its time translate by $t$, then the modular group $\Delta_{\mathcal{N}}^{\mathrm{i} u}$ of $\mathcal{N}$ transforms $\mathcal{N}(t)$ into $\mathcal{N}(\varphi(u, t))$ with a certain function $\varphi(u, t)$. Likewise, the group with a positive generator transforms $\mathcal{N}(t)$ into $\mathcal{N}(\psi(\tau, t))$, where $\psi$ is another function of $t$ and the group parameter $\tau$. The precise statements are given in Theorem 2.1. We also discuss the action of $\Delta_{\mathcal{N}}^{\mathrm{i} u}$ on individual observables in $\mathcal{N}(t)$ for $t$ large and show that, in a sense made precise in Theorems 2.2 and 2.3, this action approximates a time translation by $-\beta u$ as $t / \beta \rightarrow \infty$.

In Section 3 we consider two dimensional models that factorize in the light cone coordinates. Applying the results of the previous section to the algebras on each of the light rays one obtains a geometric description of the actions of the groups associated with the forward light cone and a space like wedge. In the case of the forward light cone, the 
algebra of a translated light cone is mapped into another such algebra. An analogous statement holds for the wedge. The flow patterns are illustrated in Figs. 1-2. Close to the apex of the light cone and the edge of the wedge the actions of the modular flow is essentially the same as for the zero temperature case, i.e., dilations for the forward light cone and Lorentz boosts for the wedge. On the other hand, at large distances from the domain boundary compared to the inverse temperature the modular flow approaches the dynamical flow, i.e., the time translations.

The one parameter unitary group with positive generator associated with the forward light cone, which in the limiting case of zero temperature reduces to time translations, approximates the dynamical flow close to the apex of the light cone. It corresponds everywhere to a decelerated movement towards the origin in the space variable (Fig. 3). Formally at least, this may be regarded as a reverse Unruh-Effect [U76], [Sew80], [Sew82]: In the latter the vacuum appears as a KMS state with respect to a dynamics that accelerates points towards light-like infinity, here a KMS state appears as a vacuum with respect to a dynamics that moves points from light-like infinity towards the origin of space.

For the wedge there is also a unitary group with positive generator which has the KMS state as a ground state. This group operates on the observables for a restricted parameter range. It approximates the time translations close to the space axis and light like translations far away from the space axis. The action of this group is illustrated in Fig. 4. This action may also be interpreted as a kind of reverse Unruh effect, because the acceleration is here away from the wedge, whereas in the usual Unruh effect the acceleration points in the direction of the wedge.

In Section 4 we compute explicitly the modular groups and the groups with positive generator for a quasi free KMS state on the Weyl algebra of a generalized free field in 2D space time that factorizes in light cone coordinates. For a field of minimal scaling dimension one obtains a strengthening of the general results of the previous section on the group actions: A local algebra $\mathcal{M}(\mathcal{O})$ with $\mathcal{O}$ a double cone is transformed into an algebra of the same kind. For fields of higher scaling dimension, however, double cone localization may be get lost under the group action and only a localization in a translated light cone or wedge remains.

\section{The group generated by translations and the modular action}

Let $\left(\mathcal{A}, \alpha_{t}\right)$ be a $C^{*}$-dynamical system and $\mathcal{B}$ a subalgebra, such that

$$
\alpha_{t} \mathcal{B} \subset \mathcal{B} \quad \text { for } t \geq 0 \text {. }
$$

Suppose furthermore that the algebra $\cup_{t \in \mathbf{R}} \alpha_{t} \mathcal{B}$ is norm dense in $\mathcal{A}$. Let $\omega$ be a KMS state [BR79] for the dynamical system $\left(\mathcal{A}, \alpha_{t}\right)$ at inverse temperature $\beta$ and denote by $\pi$ the corresponding GNS representation of $\mathcal{A}$ with cyclic vector $\Omega$, and by $T(t)$ the unitary implementation of $\alpha_{t}$ on the GNS Hilbert space $\mathcal{H}$. Put $\mathcal{M}=\pi(\mathcal{A})^{\prime \prime}$ and $\mathcal{N}=\pi(\mathcal{B})^{\prime \prime}$.

Because of the analyticity properties of the time translations in a KMS state the vector $\Omega$ is separating for $\mathcal{M}$ and hence also for $\mathcal{N}$. Moreover, $\Omega$ is cyclic for $\mathcal{M}$ (by definition) and since $\cup_{t \in \mathbf{R}} \alpha_{t} \mathcal{B}$ is dense in $\mathcal{A}$ it follows by a Reeh-Schlieder type argument that $\Omega$ is cyclic for $\mathcal{N}$ also. 
Let $\Delta_{\mathcal{M}}$ and $J_{\mathcal{M}}$ be the modular objects corresponding to $\Omega$ and $\mathcal{M}$. We have

$$
\Delta_{\mathcal{M}}^{\mathrm{i} s}=T(-\beta s)
$$

where the sign is a consequence of different conventions in physics and mathematics: For $A \in \mathcal{M}$ the expression $T(t) A \Omega$ has an analytic continuation into the strip $S(0, \beta / 2)$, where

$$
S(a, b):=\{z \in \mathbb{C}: a<\operatorname{Im} z<b\},
$$

while $\Delta_{\mathcal{M}}^{\mathrm{is}} A \Omega$ has an analytic continuation into $S(-1 / 2,0)$, by the sign convention in modular theory. Since $J_{\mathcal{M}} \Delta_{\mathcal{M}}^{1 / 2} A \Omega=A^{*} \Omega$, it follows from (2.2) that

$$
T(t+i \beta) A \Omega=J_{\mathcal{M}} T(t) A^{*} \Omega .
$$

By assumption (2.1) we have

$$
T(t) \mathcal{N} T(-t) \subset \mathcal{N} \quad \text { for } t \geq 0,
$$

i.e., we are in the situation of a half-sided translation in the sense of [Bch92]. Because of (2.2) we are also in the situation of a half-sided modular inclusion in the sense of Wiesbrock [Wie93], i.e.,

$$
\Delta_{\mathcal{M}}^{\mathrm{i} s} \mathcal{N} \Delta_{\mathcal{M}}^{-\mathrm{i} s} \subset \mathcal{N} \quad \text { for } s \leq 0
$$

If $T(t)$ had a positive generator, then (2.5) would imply the well known relations [Bch92] between $T(t)$ and the modular group $\Delta_{\mathcal{N}}^{\mathrm{i} u}$. In a KMS state, however, the spectrum of the Hamiltonian is the whole real axis and the analysis of [Bch92] has to be generalized. The main results of this generalization are stated in Eqs. (2.20), (2.29) and (2.31) below.

We start with a heuristic discussion of the consequences of (2.6), similar to that in [Wie93]. This discussion disregards questions of domains of unbounded operators, but it leads quickly to the commutation relations between $\Delta_{\mathcal{M}}^{\mathrm{i} s}$ and $\Delta_{\mathcal{N}}^{\mathrm{i} u}$ stated in [Wie97]. A rigorous proof of these relations follows from the results of [Bch95] and will be given after the discussion.

Since $\mathcal{N} \subset \mathcal{M}$ it follows by standard arguments that $\Delta_{\mathcal{N}} \geq \Delta_{\mathcal{M}}$ and this, domain questions aside, implies that

$$
G:=\log \Delta_{\mathcal{N}}-\log \Delta_{\mathcal{M}}
$$

is a non-negative operator, because log is an operator monotone function. Eqs. (2.1), (2.2), (2.6) and the Trotter product formula now lead to

$$
e^{\mathrm{i} \tau G} \mathcal{N} e^{-\mathrm{i} \tau G} \subset \mathcal{N} \quad \text { for } \tau \geq 0
$$

Putting $U(\tau):=\exp (\mathrm{i} \tau G)$, Eq. (2.8) and $G \geq 0$ imply [Bch92]

$$
\Delta_{\mathcal{N}}^{\mathrm{i} u} U(\tau) \Delta_{\mathcal{N}}^{-\mathrm{i} u}=U\left(e^{-2 \pi u} \tau\right)
$$

for all $\tau, u \in \mathbf{R}$. Hence we obtain a unitary representation of the two parameter Lie group $\mathcal{G}$ with elements $(\tau, u) \in \mathbf{R}^{2}$ and the composition law

$$
(\tau, u) \circ\left(\tau^{\prime}, u^{\prime}\right)=\left(\tau+e^{-2 \pi u} \tau^{\prime}, u+u^{\prime}\right) .
$$


The representation $U(\tau, u)$ corresponding to $(2.9)$ is

$$
U(\tau, u):=e^{\mathrm{i} \tau G} \Delta_{\mathcal{N}}^{\mathrm{i} u}
$$

The group $\mathcal{G}$ defined by (2.10) is the semidirect product of $\mathbf{R}$ with itself and is the unique two dimensional non-abelian Lie group (" $a x+b$-group"). Some of its properties are discussed in [Bch98].

For a discussion of the one parameter subgroups and the Lie algebra of $\mathcal{G}$ it is convenient to realize the group in terms of $2 \times 2$ matrices:

$$
(\tau, u) \leftrightarrow\left(\begin{array}{ll}
1 & \tau \\
0 & 1
\end{array}\right) \cdot\left(\begin{array}{cc}
e^{-2 \pi u} & 0 \\
0 & 1
\end{array}\right)=\left(\begin{array}{cc}
e^{-2 \pi u} & \tau \\
0 & 1
\end{array}\right)
$$

It is straightforward to determine the one parameter subgroups, $r \mapsto g(r)$ of $\mathcal{G}$. These have the form

$$
g_{a, b}(r)=\left(\begin{array}{cc}
e^{a r} & \frac{b}{a}\left(e^{a r}-1\right) \\
0 & 1
\end{array}\right)
$$

with $a, b \in \mathbf{R}$. In the half-plane $\left(\tau, e^{-2 \pi u}\right) \in \mathbf{R} \times \mathbf{R}_{+}$these correspond to straight lines through $(0,1)$. The infinitesimal generator of $g_{a, b}(r)$ is

$$
\hat{g}_{a, b}=\left.\frac{d}{d r} g_{a, b}(r)\right|_{r=0}=\left(\begin{array}{ll}
a & b \\
0 & 0
\end{array}\right) .
$$

The group $\Delta_{\mathcal{N}}^{\mathrm{i} u}$ corresponds to $a=-2 \pi, b=0$; the group $\exp (i \tau G)$ to $a=0, b=1$. Since the generator of $\Delta_{\mathcal{M}}^{\text {is }}$ is $\log \Delta_{\mathcal{N}}-G$, this one parameter group corresponds to $a=-2 \pi$, $b=-1$. Denoting for short the one parameter subgroups of $\mathcal{G}$ in these three cases by $g_{\mathcal{N}}(u), g_{\text {pos }}(\tau)$ and $g_{\mathcal{M}}(s)$ respectively, we have

$$
g_{\mathcal{N}}(u)=\left(\begin{array}{cc}
e^{-2 \pi u} & 0 \\
0 & 1
\end{array}\right), \quad g_{\mathrm{pos}}(\tau)=\left(\begin{array}{ll}
1 & \tau \\
0 & 1
\end{array}\right), \quad g_{\mathcal{M}}(s)=\left(\begin{array}{cc}
e^{-2 \pi s} & \frac{1}{2 \pi}\left(e^{-2 \pi s}-1\right) \\
0 & 1
\end{array}\right)
$$

One verifies the relation

$$
g_{\mathcal{N}}(u) \cdot g_{\mathcal{M}}(s)=g_{\mathcal{M}}(F(u, s)) \cdot g_{\mathcal{N}}(-F(u, s)+s+u)
$$

with

$$
F(u, s)=-\frac{1}{2 \pi} \log \left\{1+e^{-2 \pi u}\left(e^{-2 \pi s}-1\right)\right\},
$$

provided

$$
1+e^{-2 \pi u}\left(e^{-2 \pi s}-1\right)>0
$$

which is always fulfilled for $s \leq 0$. The relation corresponding to (2.16) for the modular $\operatorname{groups} \Delta_{\mathcal{N}}^{\mathrm{i} u}$ and $\Delta_{\mathcal{M}}^{\mathrm{i} s}$ is

$$
\Delta_{\mathcal{N}}^{\mathrm{i} u} \cdot \Delta_{\mathcal{M}}^{\mathrm{i} s}=\Delta_{\mathcal{M}}^{\mathrm{i} F(u, s)} \cdot \Delta_{\mathcal{N}}^{\mathrm{i}(-F(u, s)+s+u)}
$$


This relation appears also in [Wie97]; our heuristic discussion has brought its group theoretical origin into focus.

In terms of the original translation group $T(t)$ we can, because of $(2.2)$, write $(2.19)$ as

$$
\Delta_{\mathcal{N}}^{\mathrm{i} u} \cdot T(t) \cdot \Delta_{\mathcal{N}}^{-\mathrm{i} u}=T\left(\frac{\beta}{2 \pi} \log \left\{1+e^{-2 \pi u}\left(e^{2 \pi t / \beta}-1\right)\right\}\right) \cdot \Delta_{\mathcal{N}}^{\mathrm{i} \frac{1}{2 \pi} \log \left\{1+e^{-2 \pi u}\left(e^{2 \pi t / \beta}-1\right)\right\}-\mathrm{i} \frac{t}{\beta}} .
$$

In the limit $\beta \rightarrow \infty$ we recover the Bisognano-Wichmann result

$$
\Delta_{\mathcal{N}}^{\mathrm{i} u} \cdot T(t) \cdot \Delta_{\mathcal{N}}^{-\mathrm{i} u}=T\left(e^{-2 \pi u} t\right)
$$

The one parameter groups $g_{a, b}(r)$ can be expressed in terms of $g_{\mathcal{M}}(s)$ and $g_{\mathcal{N}}(u)$ :

$$
g_{a, b}(r)=g_{\mathcal{M}}(s(r)) \cdot g_{\mathcal{N}}(u(r))=g_{\mathcal{N}}(u(-r)) \cdot g_{\mathcal{M}}(s(-r))
$$

with

$$
\begin{aligned}
& s(r)=-\frac{1}{2 \pi} \log \left\{1+\frac{2 \pi b}{a}\left(e^{a r}-1\right)\right\} \\
& u(r)=-s(r)-\frac{a}{2 \pi} r .
\end{aligned}
$$

Specializing to $a=0, b=1$ we obtain for $\tau>-1 /(2 \pi)$

$$
g_{\mathrm{pos}}(\tau)=g_{\mathcal{M}}\left(-(2 \pi)^{-1} \log (1+2 \pi \tau)\right) \cdot g_{\mathcal{N}}\left((2 \pi)^{-1} \log (1+2 \pi \tau)\right)
$$

and hence

$$
U(\tau)=\Delta_{\mathcal{M}}^{-\frac{\mathrm{i}}{2 \pi} \log (1+2 \pi \tau)} \cdot \Delta_{\mathcal{N}}^{\frac{\mathrm{i}}{2 \pi} \log (1+2 \pi \tau)}=\Delta_{\mathcal{N}}^{-\frac{\mathrm{i}}{2 \pi} \log (1-2 \pi \tau)} \cdot \Delta_{\mathcal{M}}^{\frac{\mathrm{i}}{2 \pi} \log (1-2 \pi \tau)} .
$$

The first representation can be used for $\tau>-1 /(2 \pi)$, the second one for $\tau<1 /(2 \pi)$.

The group $g_{\mathcal{N}}(u)$ operates on $g_{\text {pos }}(\tau)$ according to

$$
g_{\mathcal{N}}(u) g_{\mathrm{pos}}(\tau) g_{\mathcal{N}}(-u)=g_{\mathrm{pos}}(\exp (-2 \pi u) \tau)
$$

which is just the abstract form of the basic relation (2.9). This is a special case of the general relation

$$
g_{a, b}(r) g_{\mathrm{pos}}(\tau) g_{a, b}(-r)=g_{\mathrm{pos}}(\exp (a r) \tau)
$$

For $a=-2 \pi, b=-1$, i.e. $g_{\mathcal{M}}$, the corresponding relation for the unitary groups on Hilbert space is

$$
\Delta_{\mathcal{M}}^{\mathrm{i} s} U(\tau) \Delta_{\mathcal{M}}^{-\mathrm{i} s}=U(\exp (-2 \pi s) \tau)
$$

which follows also directly from (2.25) and (2.19). We note in passing that (2.28) may be interpreted as an "Anosov relation" that leads to exponential clustering of matrix elements of the time translations $T(t)=\Delta_{\mathcal{M}}^{-\mathrm{i} t / \beta}$ in states of the form $A \Omega$ with $A$ in a dense subalgebra of $\mathcal{M}$ [ENTS95]. 
Defining $\Gamma(\tau):=U(\tau / \beta)$ we have by $(2.25)$

$$
\begin{aligned}
\Gamma(\tau) & =T\left(\frac{\beta}{2 \pi} \log \{1+(2 \pi \tau / \beta)\}\right) \cdot \Delta_{\mathcal{N}}^{\frac{i}{2 \pi}} \log \{1+(2 \pi \tau / \beta)\} \\
& =\Delta_{\mathcal{N}}^{-\frac{i}{2 \pi} \log \{1-(2 \pi \tau / \beta)\}} \cdot T\left(-\frac{\beta}{2 \pi} \log \{1-(2 \pi \tau / \beta)\}\right)
\end{aligned}
$$

where the first equality is valid for $\tau>-\beta /(2 \pi)$ and the second for $\tau<\beta /(2 \pi)$. Evidently $\Gamma(\tau) \rightarrow T(\tau)$ for $\beta \rightarrow \infty$, and

$$
G / \beta=H+\frac{1}{\beta} \log \Delta_{\mathcal{N}}
$$

tends in this limit to the Hamiltonian $H$, which in the vacuum representation is $\geq 0$.

The relation (2.28) means that

$$
T(t) \Gamma(\tau) T(-t)=\Gamma(\exp (2 \pi t / \beta) \tau)
$$

By (2.8) and our assumption that $\cup_{t} \alpha_{t} \mathcal{B}$ is norm dense in $\mathcal{A}$ (and hence $\cup_{t}$ ad $T(t) \mathcal{N}$ weakly dense in $\mathcal{M}$ ), we may thus conclude that

$$
\operatorname{ad} \Gamma(\tau) \mathcal{M} \subset \mathcal{M} \quad \text { for all } \tau \geq 0
$$

A rigorous proof of the relations (2.19) and (2.25) (and hence of (2.20), (2.29) and (2.31)) can be obtained by applying Theorems A and B in [Bch95] to the operator valued functions

$$
V(v)=\Delta_{\mathcal{M}}^{-\mathrm{i} v} \Delta_{\mathcal{N}}^{\mathrm{i} v}
$$

and $W(w)=V(v(w))$, where

$$
v(w)=\frac{1}{2 \pi} \log \left(1+e^{2 \pi w}\right) .
$$

The function $V(v)$ has a bounded analytic continuation into the strip $S(0,1 / 2)$ with continuous boundary values and satisfies the relation

$$
V\left(v+\frac{\mathrm{i}}{2}\right)=J_{\mathcal{M}} V(v) J_{\mathcal{N}}
$$

for $v \in \mathbf{R}$. Moreover, ad $V(v)$ maps $\mathcal{N}$ into $\mathcal{N}$ for $v \geq 0$ and the commutant $\mathcal{N}^{\prime}$ into $\mathcal{N}^{\prime}$ for $v \leq 0$. By (2.35) it follows that ad $V\left(v+\frac{\mathrm{i}}{2}\right)$ maps $\mathcal{N}^{\prime}$ into $\mathcal{N}^{\prime}$ for all $v$.

In order to apply Theorem B in [Bch95] we have to map the strip $S(0,1 / 2)$ biholomorphically onto itself in such a way that $\mathbf{R}$ is mapped onto $\mathbf{R}_{+}$and $\mathbf{R}+\frac{\mathrm{i}}{2}$ onto $\left(\mathbf{R}+\frac{\mathrm{i}}{2}\right) \cup \mathbf{R}_{-}$. The map (2.34) accomplishes this. It has a singularity at $w=\mathrm{i} / 2$, but as remarked in [Bch95] such a singularity is harmless. 
[The reason is as follows: Theorem B in [Bch95] is based on the edge-of-the-wedge theorem, applied to matrix elements of the operator valued function

$$
(u, w) \mapsto \Delta_{\mathcal{N}}^{\mathrm{i} u} W(w) \Delta_{\mathcal{N}}^{-\mathrm{i} u}
$$

These matrix elements have bounded analytic continuations, which are continuous at the boundary of their domain with the possible exception of points with $w=\mathrm{i} / 2$. By the dominated convergence theorem and the boundedness of (2.36) this piece-wise continuity is sufficient to ensure coincidence of boundary values in the sense of distributions. The edge-of-the-wedge theorem then implies analyticity in the coincidence region, so continuity in the points with $w=\mathrm{i} / 2$ holds a fortiori.]

Theorem B in [Bch95] leads to the general relations

$$
\Delta_{\mathcal{N}}^{\mathrm{i} u} W(w) \Delta_{\mathcal{N}}^{-\mathrm{i} u}=W(w-u)
$$

and

$$
J_{\mathcal{N}} W(w) J_{\mathcal{N}}=W\left(w+\frac{\mathrm{i}}{2}\right)
$$

Eq. (2.37) is precisely (2.19) in case (2.18) holds, but note that (2.37) is true for all $u, w \in \mathbf{R}$. As noted by Wiesbrock ([Wie93], [Wie97]) these relations imply that $\Delta_{\mathcal{N}}^{\mathrm{i} u}$ and $\Delta_{\mathcal{M}}^{\text {is }}$ generate a unitary representation of the Lie group $\mathcal{G}$. The infinitesimal generators $\log \Delta_{\mathcal{N}}$ and $\log \Delta_{\mathcal{M}}$, together with their real linear combinations, are thus essentially self adjoint on a common core. The representation $U(\tau)$ of the one parameter subgroup $g_{\text {pos }}(\tau)$ fulfills together with $\Delta_{\mathcal{N}}^{\mathrm{i} u}$ the relation $(2.9)$ (because of the corresponding relation in $\mathcal{G}$ ), and this implies by [Wie92] that $U(\tau)=\exp (\mathrm{i} \tau G)$ with $G \geq 0$. Hence the starting point of the heuristic discussion is rigorously justified.

The following theorem summarizes the main conclusions of the preceding discussion and states in addition the most important consequence of the relations (2.20), (2.29) and (2.31) for the present investigation, namely the action of the group $\mathcal{G}$ on translates of $\mathcal{N}$.

\subsection{THEOREM:}

Let $\left(\mathcal{A}, \alpha_{t}\right)$ be a $C^{*}$-dynamical system and $\mathcal{B}$ a subalgebra such that $\alpha_{t} \mathcal{B} \subset \mathcal{B}$ for $t \geq 0$ and $\cup_{t} \alpha_{t} \mathcal{B}$ is norm dense in $\mathcal{A}$. In the GNS representation defined by a KMS state on $\mathcal{A}$ at inverse temperature $\beta$ let $\mathcal{M}$ and $\mathcal{N}$ denote the weak closures of $\pi(\mathcal{A})$ and $\pi(\mathcal{B})$ respectively, and $T(t)=\exp (\mathrm{it} H)$ the unitary group implementing $\alpha_{t}$. Denote $\operatorname{ad} T(t) \mathcal{N}=\mathcal{N}(t)$. Then

(i) The translations $T(t)$ and the modular group $\Delta_{\mathcal{N}}^{\mathrm{i} u}$, defined by $\mathcal{N}$ and the KMS state vector, fulfill the relation (2.20). We have

$$
\operatorname{ad} \Delta_{\mathcal{N}}^{\mathrm{i} u} \mathcal{N}(t)=\mathcal{N}(\varphi(u, t))
$$

with

$$
\varphi(u, t)=\frac{\beta}{2 \pi} \log \left\{1+\mathrm{e}^{-2 \pi u}\left(\mathrm{e}^{2 \pi t / \beta}-1\right)\right\}
$$

for all $u, t$ satisfying

$$
1+\mathrm{e}^{-2 \pi u}\left(\mathrm{e}^{2 \pi t / \beta}-1\right)>0 .
$$


In particular,

$$
\operatorname{ad} \Delta_{\mathcal{N}}^{\mathrm{i} u} \mathcal{M} \subset \mathcal{M}
$$

for all $u \geq 0$, and

$$
\mathcal{N}=\bigcap_{u \geq 0} \operatorname{ad} \Delta_{\mathcal{N}}^{\mathrm{i} u} \mathcal{M}
$$

(ii) The operator $G=\beta H+\log \Delta_{\mathcal{N}}$ is non-negative and essentially self adjoint on a common core of $H$ and $\log \Delta_{N}$. The one parameter group $\Gamma(\tau)=\exp (\mathrm{i} \tau G / \beta)$ is given by (2.29) and the groups $\Gamma(\tau)$ and $T(t)$ satisfy (2.31). We have

$$
\operatorname{ad} \Gamma(\tau) \mathcal{N}(t)=\mathcal{N}(\psi(u, t))
$$

with

$$
\psi(\tau, t)=t+\frac{\beta}{2 \pi} \log \left\{1+\frac{2 \pi \tau}{\beta} \mathrm{e}^{-2 \pi t / \beta}\right\}
$$

for all $\tau, t$ satisfying

$$
1+\frac{2 \pi \tau}{\beta} \mathrm{e}^{-2 \pi t / \beta}>0
$$

In particular,

$$
\operatorname{ad} \Gamma(\tau) \mathcal{M} \subset \mathcal{M} \quad \text { and } \quad \operatorname{ad} \Gamma(\tau) \mathcal{N} \subset \mathcal{N}
$$

for $\tau \geq 0$, and

$$
\mathcal{N}=\operatorname{ad} \Gamma(\beta / 2 \pi) \mathcal{M}
$$

Proof: As already noted, the key relations (2.20), (2.29) and (2.31), and the self adjointness and positivity of $G$ are a rigorous consequence of the Theorems in [Bch95], [Wie92], [Wie93], and [Wie97]. Eqs. (2.39) and (2.44) follow directly from (2.20) and (2.29) and the fact that $\varphi(-u, \varphi(u, t))=t, \psi(-\tau, \psi(\tau, t))=t$ for $(u, t)$ and $(\tau, t)$ satisfying $(2.41)$ and $(2.46)$ respectively. Eqs. (2.42), (2.43), (2.47) and (2.48) are simple consequences of (2.39) and (2.44) since $\cup_{t}$ ad $T(t) \mathcal{N}$ is dense in $\mathcal{M}$.

As a last topic in this section we discuss the relation between the translation group $T(t)$ and the modular group $\Delta_{\mathcal{N}}^{\mathrm{i} u}$. Since $T(-\beta u)=\Delta_{\mathcal{M}}^{\mathrm{i} u}$, one may expect that the actions of $T(-\beta u)$ and $\Delta_{\mathcal{N}}^{\mathrm{i} u}$ approximately coincide on elements that have been translated far into $\mathcal{N}$, so that "boundary effects" are negligible. That this intuition is indeed solidly founded is the content of the next two theorems. The first concerns certain matrix elements of the unitary groups, and gives an estimate for the rate of the convergence. The second is about strong convergence of Hilbert space vectors and operators, but the error estimates are less explicit.

\subsection{THEOREM:}

If $A \in \mathcal{N}(t)$ and $B \in \mathcal{N}^{\prime}$ the following estimate holds for $t>0$ and all $u$ :

$$
\left|\left(B \Omega, \Delta_{\mathcal{N}}^{\mathrm{i} u} A \Omega\right)-(B \Omega, T(-\beta u) A \Omega)\right| \leq 2 M \min \left\{\frac{|\exp (2 \pi u)-1|}{\exp (2 \pi t / \beta)-1}, 1\right\}
$$


with

$$
M=\max \left\{\|A \Omega\|\|B \Omega\|,\left\|A^{*} \Omega\right\|\left\|B^{*} \Omega\right\|\right\} .
$$

Proof: Consider the two functions

$$
F^{+}(u)=\left(B \Omega, \Delta_{\mathcal{N}}^{-\mathrm{i} u} T(-\beta u) A \Omega\right), \quad \text { and } \quad F^{-}(u)=\left(A^{*} \Omega, T(\beta u) \Delta_{\mathcal{N}}^{\mathrm{i} u} B^{*} \Omega\right) .
$$

Theorem A in [Bch95] implies that $\Delta_{\mathcal{N}}^{-\mathrm{i} u} T(-\beta u)$ has a bounded analytic continuation into the strip $S\left(-\frac{1}{2}, 0\right)$. It follows that $F^{+}$has an analytic continuation into $S\left(-\frac{1}{2}, 0\right)$, and $F^{-}$ into $S\left(0, \frac{1}{2}\right)$. Moreover, by continuity of the unitary groups, $F^{ \pm}$is continuous on the real axis.

Denoting $j_{\mathcal{M}}=\operatorname{ad} J_{\mathcal{M}}, j_{\mathcal{N}}=\operatorname{ad} J_{\mathcal{N}}$ we obtain

$$
\begin{aligned}
& F^{+}\left(u-\frac{\mathrm{i}}{2}\right)=\left(B \Omega, J_{\mathcal{N}} \Delta_{\mathcal{N}}^{-\mathrm{i} u} T(-\beta u) J_{\mathcal{M}} A \Omega\right)=\left(\Delta_{\mathcal{N}}^{-\mathrm{i} u} T(-\beta u) j_{\mathcal{M}}(A) \Omega, j_{\mathcal{N}}(B) \Omega\right), \\
& F^{-}\left(u+\frac{\mathrm{i}}{2}\right)=\left(A^{*} \Omega, J_{\mathcal{M}} T(\beta u) \Delta_{\mathcal{N}}^{\mathrm{i} u} J_{\mathcal{N}} B^{*} \Omega\right)=\left(T(\beta u) \Delta_{\mathcal{N}}^{\mathrm{i} u} j_{\mathcal{N}}\left(B^{*}\right) \Omega, j_{\mathcal{M}}\left(A^{*}\right) \Omega\right) .
\end{aligned}
$$

In particular $F^{ \pm}$is continuous at $u \pm \mathrm{i} / 2, u \in \mathbf{R}$, and $j_{\mathcal{N}}\left(B^{*}\right) \in \mathcal{N}$ and $j_{\mathcal{M}}\left(A^{*}\right) \in \mathcal{M}^{\prime}$ implies

$$
F^{+}\left(u-\frac{\mathrm{i}}{2}\right)=F^{-}\left(u+\frac{\mathrm{i}}{2}\right)
$$

Moreover, since $T(-s) A T(s) \in \mathcal{N}$ for $s<t$, we have

$$
F^{+}(u)=F^{-}(u) \text { for } u<t / \beta .
$$

Hence $F^{+}$and $F^{-}$have a common analytic continuation to a periodic function, $F$, with the period $\mathrm{i}$ and cuts $[t / \beta, \infty)+\mathrm{i} n, n \in \mathbf{Z}$. This function is majorized by

$$
M=\max \left\{\|A \Omega\|\|B \Omega\|,\left\|A^{*} \Omega\right\|\left\|B^{*} \Omega\right\|\right\} .
$$

The function $F(z)-F(0)$ vanishes at $z=\mathrm{i} n, n \in \mathbf{Z}$, and is bounded by $2 M$. Therefore,

$$
G(z)=\frac{F(z)-F(0)}{\exp (2 \pi z)-1}
$$

is analytic and bounded in the same domain as $F$. Along the cuts we have $|G(z)| \leq$ $2 M(\exp (2 \pi t / \beta)-1)^{-1}$. By the maximum modulus principle this estimate holds everywhere and thus

$$
\left|\left(B \Omega, \Delta_{\mathcal{N}}^{-\mathrm{i} u} T(-\beta u) A \Omega\right)-(B \Omega, A \Omega)\right| \leq 2 M \frac{|\exp (2 \pi u)-1|}{\exp (2 \pi t / \beta)-1} .
$$

This estimate blows up for $t \rightarrow 0$, but the left-hand side is trivially bounded by $2 M$ for all real $u$ and $t$. Replacing $B \in \mathcal{N}^{\prime}$ by ad $\Delta_{\mathcal{N}}^{-\mathrm{i} u} B \in \mathcal{N}^{\prime}$ does not change $M$, so (2.55) gives the desired estimate (2.49). 


\subsection{THEOREM:}

(i) For every $A \in \mathcal{M}$ and Hilbert space vector $\Psi$

$$
\lim _{t \rightarrow \infty}\left\|\Delta_{\mathcal{N}}^{\mathrm{i} u} A(t) \Psi-T(-\beta u) A(t) \Psi\right\|=0
$$

with $A(t)=\operatorname{ad} T(t) A$. The convergence is uniform on half sided $u$-intervals $I=\left(-\infty, u_{0}\right]$, $u_{0}<\infty$.

(ii) For every $A$ in a dense subalgebra of $\mathcal{M}$

$$
\lim _{t \rightarrow \infty}\left\|\operatorname{ad} \Delta_{\mathcal{N}}^{\mathrm{i} u} A(t)-\operatorname{ad} T(-\beta u) A(t)\right\|=0
$$

with uniform convergence on half sided $u$-intervals.

Proof: From (2.48), (2.2) and (2.31) it follows that

$$
\Delta_{\mathcal{N}}^{\mathrm{i} u}=\Gamma(\beta / 2 \pi) T(-\beta u) \Gamma(-\beta / 2 \pi)=T(-\beta u) \Gamma((\exp (2 \pi u)-1) \beta / 2 \pi)
$$

Hence, using (2.31) again,

$$
\operatorname{ad} \Delta_{\mathcal{N}}^{\mathrm{i} u} A(t)-\operatorname{ad} T(-\beta u) A(t)=\operatorname{ad} T(t-\beta u)[\operatorname{ad} \Gamma(\exp (-2 \pi t / \beta) h(u)) A-A]
$$

with $h(u)=(\exp (2 \pi u)-1) \beta / 2 \pi$. Now (i) follows from the strong convergence of $\Gamma(\tau)$ to 1 as $\tau \rightarrow 0$, because $\sup _{u \in I}|h(u)|<\infty$ for $I=\left(-\infty, u_{0}\right]$.

For general $A \in \mathcal{M},\|\operatorname{ad} \Gamma(\tau) A-A\|$ need not converge to zero as $\tau \rightarrow 0$. However, on elements of the form $A_{g}=\int g(\tau) \operatorname{ad} \Gamma(\tau) A d \tau$ with $g$ continuous of compact support, this convergence holds. Moreover, if $g$ is continuously differentiable, then (2.69) implies

$$
\left\|\operatorname{ad} \Delta_{\mathcal{N}}^{\mathrm{i} u} A_{g}(t)-\operatorname{ad} T(-\beta u) A_{g}(t)\right\| \leq\|A\| \cdot\|d g / d \tau\|_{1} \cdot \sup _{u \in I}|h(u)| \cdot e^{-2 \pi t / \beta} .
$$

By (2.44) such regularized elements are dense in $\mathcal{M}$ if the support of $g$ is sufficiently small, and $A_{g} \rightarrow A$ weakly if $g$ tends to a delta function.

\section{Two dimensional models}

The general results of the preceding setting were formulated for a $C^{*}$-dynamical system $\left(\mathcal{A}, \alpha_{t}\right)$ and a subalgebra $\mathcal{B}$, invariant under half-sided shifts by $\alpha_{t}$. We shall now be more specific and consider a quasi-local algebra $\mathcal{A}$ generated by a local net $\mathcal{O} \mapsto \mathcal{A}(\mathcal{O})$ of $C^{*}$ algebras and $\mathcal{B}=\mathcal{A}\left(\mathcal{O}_{0}\right)$ with $\mathcal{O}_{0}$ a domain invariant under half-sided translations in the $t$-direction. In the representation $\pi$ generated by a KMS state $\omega$ we denote $\pi(\mathcal{A}(\mathcal{O}))^{\prime \prime}$ by $\mathcal{M}(\mathcal{O})$ and $\pi(\mathcal{A})^{\prime \prime}$ by $\mathcal{M}$, as before.

Eqs. (2.39) and (2.44) describe the action of the modular- and $\Gamma$-groups associated with $\mathcal{N}=\mathcal{M}\left(\mathcal{O}_{0}\right)$ on the translated algebras $\mathcal{M}\left(\mathcal{O}_{0}+t \mathbf{e}\right)$, where $\mathbf{e}$ is the unit vector in the $t$-direction. We now want to investigate how the groups associated with $M\left(\mathcal{O}_{0}\right)$ act on the 
algebras of more general domains than $\mathcal{O}_{0}+$ te, in particular $\mathcal{O}_{0}+\mathbf{x}$ with $\mathbf{x}$ an arbitrary vector in space-time. While a general answer to this question appears difficult, the previous results lead directly to a description of the action in the case of two dimensional theories that factorize in the light cone variables.

We start by considering local nets in two dimensional space time depending only on one light cone variable, i.e. nets on a light ray. With $x^{0}$ the time and $x^{1}$ the space coordinate of $\mathbf{x} \in \mathbf{R}^{2}$ the light cone variables are $x^{\mathrm{R}}=x^{0}+x^{1}$ and $x^{\mathrm{L}}=x^{0}-x^{1}$. We consider either one of them and denote it simply by $x$. Note that translations in time $t=x^{0}$ are equivalent to translations in $x$. A local algebra corresponding to an $x$-interval $I \subset \mathbf{R}$ is denoted by $\mathcal{M}(I)$. Local commutativity means that $\mathcal{M}\left(I_{1}\right)$ and $\mathcal{M}\left(I_{2}\right)$ commute if $I_{1} \cap I_{2}=\emptyset$.

We denote the modular group for the algebra $\mathcal{M}\left(\mathbf{R}_{+}\right)$by $\Delta_{+}^{\mathrm{i} u}$ and the corresponding group with the positive generator $G_{+} / \beta=H+(1 / \beta) \log \Delta_{+}$by $\Gamma_{+}(\tau)$. We shall also consider the algebra of the negative half axis, $\mathcal{M}\left(\mathbf{R}_{-}\right)$, with modular group $\Delta_{-}^{\mathrm{i} u}$ and the positive operator $G_{-} / \beta=H+(1 / \beta) \log \Delta_{-}$, which generates the group $\Gamma_{-}(\tau)=$ $\exp \left(\mathrm{i} \tau G_{-} / \beta\right)$. Note that ad $\Gamma_{-}(\tau)$ maps $\mathcal{M}\left(\mathbf{R}_{-}\right)$into itself for $\tau \leq 0$.

By Eq. (2.39) we have

$$
\operatorname{ad} \Delta_{+}^{\mathrm{i} u} \mathcal{M}\left(\left[x, \infty[)=\mathcal{M}\left(\left[\varphi_{+}(u, x), \infty[)\right.\right.\right.\right.
$$

with

$$
\varphi_{+}(u, x)=\frac{\beta}{2 \pi} \log \left\{1+\mathrm{e}^{-2 \pi u}\left(\mathrm{e}^{2 \pi x / \beta}-1\right)\right\},
$$

for all $x, u \in \mathbf{R}$ such that

$$
1+\exp (-2 \pi u)[\exp (2 \pi x / \beta)-1]>0 .
$$

Note that (3.2) is just the function (2.40). We denote it here by $\varphi_{+}$because there is an analogous result for $\mathcal{M}\left(\mathbf{R}_{-}\right)$:

$$
\left.\left.\left.\left.\operatorname{ad} \Delta_{-}^{\mathrm{i} u} \mathcal{M}(]-\infty, x\right]\right)=\mathcal{M}(]-\infty, \varphi_{-}(u, x)\right]\right)
$$

with

$$
\varphi_{-}(u, x)=-\varphi_{+}(-u,-x)
$$

for

$$
1+\exp (2 \pi u)[\exp (-2 \pi x / \beta)-1]>0 .
$$

Likewise, from Eq. (2.44)

$$
\operatorname{ad} \Gamma_{+}(\tau) \mathcal{M}\left(\left[x, \infty[)=\mathcal{M}\left(\left[\psi_{+}(\tau, x), \infty[)\right.\right.\right.\right.
$$

with

$$
\psi_{+}(\tau, x)=x+\frac{\beta}{2 \pi} \log \left\{1+\frac{2 \pi \tau}{\beta} \mathrm{e}^{-2 \pi x / \beta}\right\}
$$

for

$$
1+(2 \pi \tau / \beta) \exp (-2 \pi x / \beta)>0,
$$


and

$$
\left.\left.\left.\left.\operatorname{ad} \Gamma_{-}(\tau) \mathcal{M}(]-\infty, x\right]\right)=\mathcal{M}(]-\infty, \psi_{-}(u, x)\right]\right)
$$

with

$$
\psi_{-}(\tau, x)=-\psi_{+}(-\tau,-x)
$$

for

$$
1-(2 \pi \tau / \beta) \exp (2 \pi x / \beta)>0 .
$$

We now turn to models in two space-time dimensions which can be written as a tensor product of one-dimensional models in the light cone variables, $x^{\mathrm{R}}=x^{0}+x^{1}$ and $x^{\mathrm{L}}=x^{0}-x^{1}$. For a domain $I_{\mathrm{L}} \times I_{\mathrm{R}} \subset \mathbf{R}^{2}$ with $I_{\mathrm{L}}$ and $I_{\mathrm{R}}$ intervals on the $x_{\mathrm{L}}$ and $x_{\mathrm{R}}$ axis respectively, the local algebra is thus

$$
\mathcal{M}\left(I_{\mathrm{L}} \times I_{\mathrm{R}}\right)=\mathcal{M}\left(I_{\mathrm{L}}\right) \otimes \mathcal{M}\left(I_{\mathrm{R}}\right)
$$

Here $\otimes$ is the von Neumann tensor product, and $I \mapsto \mathcal{M}(I)$ is a local net of von Neumann algebras over R. (For simplicity of notation we take identical nets on both axis.) In particular, we are interested in the algebras of the forward light cone

$$
\mathcal{M}\left(\mathrm{V}^{+}\right)=\mathcal{M}\left(\mathbf{R}_{+}\right) \otimes \mathcal{M}\left(\mathbf{R}_{+}\right)
$$

and the right wedge

$$
\mathcal{M}(W)=\mathcal{M}\left(\mathbf{R}_{-}\right) \otimes \mathcal{M}\left(\mathbf{R}_{+}\right) .
$$

The modular groups for these algebras and a factorizing KMS state $\omega \otimes \omega$, where $\omega$ is a KMS state for the algebra on a light ray, are

$$
\Delta_{\mathrm{V}^{+}}^{\mathrm{i} u}=\Delta_{+}^{\mathrm{i} u} \otimes \Delta_{+}^{\mathrm{i} u}
$$

and

$$
\Delta_{W}^{\mathrm{i} u}=\Delta_{-}^{\mathrm{i} u} \otimes \Delta_{+}^{\mathrm{i} u}
$$

If $\mathbf{x} \in \mathbf{R}^{2}$ we denote the translated light cone $\mathrm{V}^{+}+\mathbf{x}$ by $\mathrm{V}_{\mathbf{x}}^{+}$and the translated wedge $W+\mathbf{x}$ by $W_{\mathbf{x}}$. From Eqs. (3.1) and (3.5) we obtain

\subsection{THEOREM:}

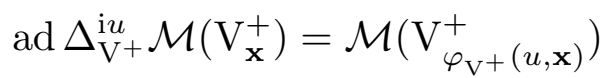

with

$$
\varphi_{\mathrm{V}^{+}}(u, \mathbf{x})=\left(\varphi_{+}\left(u, x^{\mathrm{L}}\right), \varphi_{+}\left(u, x^{\mathrm{R}}\right)\right)
$$

for $u \in \mathbf{R}$ and $\mathbf{x} \in \mathbf{R}^{2}$ such that (3.3) holds for $x=x^{\mathrm{L}}$ and $x=x^{\mathrm{R}}$. If $u \geq 0$, then ad $\Delta_{\mathrm{V}^{+}}^{\mathrm{i} u} \mathcal{M}\left(\mathrm{V}_{\mathbf{x}}^{+}\right) \subset \mathcal{M}$ for all $\mathbf{x} \in \mathbf{R}^{2}$, and if $\mathbf{x} \in \mathrm{V}^{+}$, then ad $\Delta_{\mathrm{V}^{+}}^{\mathrm{i} u} \mathcal{M}\left(\mathrm{V}_{\mathbf{x}}^{+}\right) \subset \mathcal{M}\left(\mathrm{V}^{+}\right)$for all $u$.

Likewise,

$$
\operatorname{ad} \Delta_{W}^{\mathrm{i} u} \mathcal{M}\left(W_{\mathbf{x}}\right)=\mathcal{M}\left(W_{\varphi_{W}(u, \mathbf{x})}\right)
$$


with

$$
\varphi_{W}(u, \mathbf{x})=\left(\varphi_{-}\left(u, x^{\mathrm{L}}\right), \varphi_{+}\left(u, x^{\mathrm{R}}\right)\right)
$$

for $u \in \mathbf{R}$ and $\mathbf{x} \in \mathbf{R}^{2}$ such that (3.3) holds for $x=x^{\mathrm{R}}$ and (3.6) for $x=x^{\mathrm{L}}$. If $\mathbf{x} \in W$, then $\operatorname{ad} \Delta_{W}^{\mathrm{i} u} \mathcal{M}\left(W_{\mathbf{x}}\right) \subset \mathcal{M}(W)$ for all $u$.

The flow lines of $\varphi_{\mathrm{V}^{+}}$and $\varphi_{W}$ within the respective domains are shown in Figs. 1-2.

It is evident from the figures that the character of the modular flow depends of the distance from the boundary of the domain considered (forward light cone or wedge). The natural unit of length is here the reciprocal temperature, $\beta$. Consider first the modular group of the forward ligh cone $\mathrm{V}^{+}$. In terms of the original space time coordinates $x^{0}=$ $\left(x^{\mathrm{R}}+x^{\mathrm{L}}\right) / 2$ and $x^{1}=\left(x^{\mathrm{R}}-x^{\mathrm{L}}\right) / 2$ the map $(3.19)$ takes $\left(x^{0}, x^{1}\right)$ to $\left(x^{\prime 0}, x^{\prime 1}\right)$ with

$$
x^{\prime 0}=x^{0}-\beta u+R_{\mathrm{V}^{+}}^{0}(x, u), \quad x^{1}=x^{1}+R_{\mathrm{V}^{+}}^{1}(x, u),
$$

where

$$
R_{\mathrm{V}^{+}}^{0}(x, u)=(\beta / 4 \pi) \log \left\{\left(1+e^{-2 \pi\left(x^{\mathrm{R}}-\beta u\right) / \beta}-e^{-2 \pi x^{\mathrm{R}} / \beta}\right)\left(1+e^{-2 \pi\left(x^{\mathrm{L}}-\beta u\right) / \beta}-e^{-2 \pi x^{\mathrm{L}} / \beta}\right)\right\}
$$

and

$$
R_{\mathrm{V}^{+}}^{1}(x, u)=(\beta / 4 \pi) \log \left\{\frac{1+e^{-2 \pi\left(x^{\mathrm{R}}-\beta u\right) / \beta}-e^{-2 \pi x^{\mathrm{R}} / \beta}}{1+e^{-2 \pi\left(x^{\mathrm{L}}-\beta u\right) / \beta}-e^{-2 \pi x^{\mathrm{L}} / \beta}}\right\} .
$$

Far from the domain boundary, i.e., for $x^{\mathrm{R}}, x^{\mathrm{L}}, x^{\mathrm{R}}-\beta u$ and $x^{\mathrm{L}}-\beta u$ large compared to $\beta$, the terms $R_{\mathrm{V}^{+}}^{0}$ and $R_{\mathrm{V}^{+}}^{1}$ are exponentially small, and $\psi_{\mathrm{V}^{+}}(\cdot, u)$ essentially the same as translation in time by $-\beta u$ in accord with Theorems 2.2 and 2.3. On the other hand, close to the apex of the light cone (compared to $\beta$ ), the action is essentially the same as for $\beta=\infty$, i.e., dilation by the factor $\exp (-2 \pi u)$. The deviation from a dilation is of the order $(|x| / \beta)^{2}$.

For the wedge $W$ the formulas corresponding to (3.22)-(3.24) are

$$
x^{\prime 0}=x^{0}-\beta u+R_{W}^{0}(x, u), \quad x^{1}=x^{1}+R_{W}^{1}(x, u)
$$

with

$$
R_{W}^{0}(x, u)=(\beta / 4 \pi) \log \left\{\frac{1+e^{-2 \pi\left(x^{\mathrm{R}}-\beta u\right)}-e^{-2 \pi x^{\mathrm{R}} / \beta}}{1+e^{2 \pi\left(x^{\mathrm{L}}-\beta u\right) / \beta}-e^{2 \pi x^{\mathrm{L}} / \beta}}\right\}
$$

and

$$
R_{W}^{1}(x, u)=(\beta / 4 \pi) \log \left\{\left(1+e^{-2 \pi\left(x^{\mathrm{R}}-\beta u\right)}-e^{-2 \pi x^{\mathrm{R}} / \beta}\right)\left(1+e^{2 \pi\left(x^{\mathrm{L}}-\beta u\right) / \beta}-e^{2 \pi x^{\mathrm{L}} / \beta}\right)\right\} .
$$

Note that the wedge is characterized by $x^{\mathrm{R}} \geq 0$ and $x^{\mathrm{L}} \leq 0$. Again the modular action coincides essentially with time translations far from the domain boundary. Near the edge of the wedge the coordinate $x^{\mathrm{R}}$ is scaled by $\exp (-2 \pi u)$ and $x^{\mathrm{L}}$ is scaled by $\exp (2 \pi u)$, up to terms of order $(|x| / \beta)^{2}$. This corresponds to a Lorentz boost, i.e. the modular action at temperature zero. 
From $\Gamma_{ \pm}(\tau)$ we can form the one parameter unitary groups

$$
\tau \mapsto \Gamma_{ \pm}(\tau) \otimes \Gamma_{ \pm}(\tau)
$$

on the tensor product Hilbert space. These groups have the positive generators $H+$ $(1 / \beta) \log \Delta_{ \pm, \pm}$, where $\Delta_{ \pm, \pm}=\Delta_{ \pm} \otimes 1+1 \otimes \Delta_{ \pm}$is the modular operator of $\mathcal{M}\left(\mathbf{R}_{ \pm} \times \mathbf{R}_{ \pm}\right)$. They correspond respectively to the forward and backward light cone ( ++ and --$)$ and the left and the right wedge $(+-$ and -+$)$. All four grops converge to the time translations as $\beta \rightarrow \infty$.

The group associated with the forward light cone is

$$
\Gamma_{\mathrm{V}^{+}}(\tau)=\Gamma_{+}(\tau) \otimes \Gamma_{+}(\tau)
$$

By (2.44) we have

\subsection{THEOREM:}

If $\mathbf{x} \in \mathbf{R}^{2}$ and

$$
\tau>-\beta(2 \pi)^{-1} \min \left\{e^{2 \pi x^{\mathrm{L}} / \beta}, e^{2 \pi x^{\mathrm{R}} / \beta}\right\}
$$

then

$$
\operatorname{ad} \Gamma_{\mathrm{V}^{+}}(\tau) \mathcal{M}\left(\mathrm{V}_{\mathbf{x}}^{+}\right)=\mathcal{M}\left(\mathrm{V}_{\psi_{\mathrm{V}^{+}}(\tau, \mathbf{x})}\right)
$$

with

$$
\psi_{\mathrm{V}^{+}}(\tau, \mathbf{x})=\left(\psi_{+}\left(\tau, x^{\mathrm{L}}\right), \psi_{+}\left(\tau, x^{\mathrm{R}}\right)\right)
$$

If

$$
\tau>-\beta(2 \pi)^{-1}\left(\min \left\{e^{2 \pi x^{\mathrm{L}} / \beta}, e^{2 \pi x^{\mathrm{R}} / \beta}\right\}-1\right)
$$

then

$$
\operatorname{ad} \Gamma_{\mathrm{V}^{+}}(\tau) \mathcal{M}\left(\mathrm{V}_{\mathbf{x}}^{+}\right) \subset \mathcal{M}\left(\mathrm{V}^{+}\right)
$$

The group associated with the right wedge,

$$
\Gamma_{W}(\tau)=\Gamma_{-}(\tau) \otimes \Gamma_{+}(\tau)
$$

does not induce half sided translations on the wedge algebra, but it nevertheless acts geometrically for a restricted parameter range. In fact, by Eqs. (3.7) and (3.10) we have

\subsection{THEOREM:}

If $\mathbf{x} \in \mathbf{R}^{2}$ and

$$
-\beta(2 \pi)^{-1} e^{2 \pi x^{\mathrm{R}} / \beta}<\tau<\beta(2 \pi)^{-1} e^{-2 \pi x^{\mathrm{L}} / \beta},
$$

then

$$
\operatorname{ad} \Gamma_{W}(\tau) \mathcal{M}\left(W_{\mathbf{x}}\right)=\mathcal{M}\left(W_{\psi_{W}(\tau, \mathbf{x})}\right)
$$

with

$$
\psi_{W}(\tau, \mathbf{x})=\left(\psi_{-}\left(\tau, x^{\mathrm{L}}\right), \psi_{+}\left(\tau, x^{\mathrm{R}}\right)\right)
$$


If

$$
-\beta(2 \pi)^{-1}\left(e^{2 \pi x^{\mathrm{R}} / \beta}-1\right)<\tau<\beta(2 \pi)^{-1}\left(e^{-2 \pi x^{\mathrm{L}} / \beta}-1\right)
$$

then

$$
\operatorname{ad} \Gamma_{W}(\tau) \mathcal{M}\left(W_{\mathbf{x}}\right) \subset \mathcal{M}(W)
$$

The flows of $\psi_{\mathrm{V}^{+}}$and $\psi_{W}$ are shown in Figs. 3 and 4 . The groups $\Gamma_{\mathrm{V}^{+}}(\tau)$ and $\Gamma_{W}(\tau)$, approximate the time translations close to the tip of the light cone and the edge of the wedge, respectively. Indeed, $\psi_{\mathrm{V}^{+}} \operatorname{maps}\left(x^{0}, x^{1}\right)$ to $\left(x^{\prime 0}, x^{\prime 1}\right)$ with

$$
x^{\prime 0}=x^{0}+\tau\left[\exp \left(-2 \pi x^{\mathrm{R}}\right)+\exp \left(-2 \pi x^{\mathrm{L}}\right)\right] / 2+O\left(\tau^{2} / \beta\right)
$$

and

$$
x^{\prime 1}=x^{1}+\tau\left[\exp \left(-2 \pi x^{\mathrm{R}}\right)-\exp \left(-2 \pi x^{\mathrm{L}}\right)\right] / 2+O\left(\tau^{2} / \beta\right) .
$$

For $x^{\mathrm{R}}$ and $x^{\mathrm{L}}$ both close to zero, this is close to $x^{\prime 0}=x^{0}+\tau, x^{\prime 1}=x^{1}$. More interesting, however, is the behavior of $\Gamma_{\mathrm{V}^{+}}(\tau)$ far from the apex of the cone. From Fig. 3 one sees clearly that the flow corresponds to a decelerated motion towards the origin of space. More quantitatively, the velocity $v=d x^{1} / d x^{\prime 0}$ is

$$
v=-\tanh \left(2 \pi x^{\prime 1} / \beta\right)
$$

and this differential equation has the general solution

$$
x^{\prime 0}\left(x^{1}\right)=-(\beta / 2 \pi) \log \left(\sinh \left(2 \pi x^{\prime 1} / \beta\right)\right)+C
$$

where $C$ is an arbitary constant. The path through the origin, $x^{1}=0$, corresponds formally to $C=-\infty$. The flow pattern is invariant under a shift in the time direction, in accord with (2.31).

As already mentioned in the Introduction, this flow brings points which start out with the velocity of light at infinity gradually to rest. Formally we have the reverse of an Unruh effect, for the generator of the flow of the observables is positive with the KMS state vector as a ground state. Measured in terms of the parameter $\tau$ it takes $\beta / 2 \pi \tau$-units for points to reach the forward light cone from infinity. The $\tau$-parameter along the path through the origin is related to the real time $t$ by

$$
t=(\beta / 2 \pi) \log (1+2 \pi \tau / \beta), \quad \text { i.e. } \quad \tau=(\beta / 2 \pi)(\exp (2 \pi t / \beta)-1)
$$

The $\tau$-unit is calibrated in such a way, that the $t$ - and $\tau$-scales coincide precisely where the path hits the apex of the light cone. According to Eq. (2.31) a different calibration corresponds simply to a shift of the cone in the time-direction. It is clear from (3.45) that the $\tau$-parameter is "slower" than $t$, in the sense that $d \tau / d t<1$, for a point on the path outside the light cone $(\tau<0)$, and "faster" than $t$, i.e. $d \tau / d t>1$, inside the light cone $(\tau>0)$.

For $\psi_{W}$ the equations corresponding to (3.41) and (3.42) are

$$
x^{\prime 0}=x^{0}+\tau\left[\exp \left(-2 \pi x^{\mathrm{R}}\right)+\exp \left(2 \pi x^{\mathrm{L}}\right)\right] / 2+O\left(\tau^{2} / \beta\right)
$$


and

$$
x^{\prime 1}=x^{1}+\tau\left[\exp \left(-2 \pi x^{\mathrm{R}}\right)-\exp \left(2 \pi x^{\mathrm{L}}\right)\right] / 2+O\left(\tau^{2} / \beta\right),
$$

and the velocity is

$$
v=-\tanh \left(2 \pi x^{\prime 0} / \beta\right) .
$$

Thus the velocity is small close to the space axis, but approaches \pm 1 far away from the space axis. The explicit solution of (3.48) is

$$
x^{\prime 1}\left(x^{\prime 0}\right)=-(\beta / 2 \pi) \log \left(\cosh \left(2 \pi x^{\prime 0} / \beta\right)\right)+C .
$$

This flow is invariant under a translation in the $x^{1}$-direction. The situation is here different from the light cone since not all paths pass through the wedge, and those who do, stay in the wedge only for a finite $\tau$-interval, cf. Eq. (3.39). The group even moves localized observables out of the global observable algebra in finite " $\tau$-time", cf. Eq. (3.36). The direction of acceleration is here in the opposite wedge, whereas in the usual Unruh effect it points in the direction of the wedge. In this sense we have here also a kind of reverse of the situation in the Unruh effect.

For the path passing through the origin the $\tau$-parameter is related to $t=x^{0}$ by

$$
t=\frac{\beta}{4 \pi} \log \frac{1+(2 \pi \tau / \beta)}{1-(2 \pi \tau / \beta)}, \quad \text { i.e. } \quad \tau=\frac{\beta}{2 \pi} \tanh \frac{2 \pi t}{\beta} .
$$

The relation to the proper time $t_{\mathrm{p}}$ along the path is

$$
\tau=\frac{\beta}{2 \pi} \sin \frac{2 \pi t_{\mathrm{p}}}{\beta}
$$

i.e., up to a slight deformation $\tau$ is esentially the proper time. We have

$$
d \tau / d t_{\mathrm{p}}=\cos \frac{2 \pi t_{\mathrm{p}}}{\beta}=\left(1-(2 \pi \tau / \beta)^{2}\right)^{1 / 2}
$$

so " $\tau$-time" is everywhere slower than $t_{\mathrm{p}}$ except at the origin (calibration point), where both scales coincide with $t$. A change of scale corresponds to a translation of the wedge along the $x^{1}$-axis because of $(2.31)$.

Above we have described the actions of the modular- and $\Gamma$-groups in terms of the space time coordinates $\left(x^{\mathrm{L}}, x^{\mathrm{R}}\right)$ and also in terms of $\left(x^{0}, x^{1}\right)$. The simplest description is obtained in yet another coordinate system, that is related to the others by a nonlinear transformation. For $x \in \mathbf{R}$ define

$$
\xi_{ \pm}= \pm(\beta / 2 \pi)(\exp ( \pm 2 \pi x / \beta)-1)
$$

The range of $\xi_{+}$is ] $-\beta / 2 \pi, \infty\left[\right.$ and the range of $\xi_{-}$is $]-\infty, \beta / 2 \pi\left[\right.$. With $x=x^{\mathrm{L}}$ and $x=x^{\mathrm{R}}$ respectively we thus obtain the four coordinates, $\xi_{+}^{\mathrm{L}}, \xi_{-}^{\mathrm{L}}, \xi_{+}^{\mathrm{R}}$ and $\xi_{-}^{\mathrm{R}}$. In the case of the forward light cone we pick $\left(\xi_{+}^{\mathrm{L}}, \xi_{+}^{\mathrm{R}}\right)$ and in the case of the right wedge $\left(\xi_{-}^{\mathrm{L}}, \xi_{+}^{\mathrm{R}}\right)$ as a curvelinear coordinate system on Minkowski space. In these coordinates the 
transformations $(3.19)$, (3.21) for the groups associated with the forward light cone $\mathrm{V}^{+}$ become

$$
\left(\xi_{+}^{\mathrm{L}}, \xi_{+}^{\mathrm{R}}\right) \mapsto e^{-2 \pi u}\left(\xi_{+}^{\mathrm{L}}, \xi_{+}^{\mathrm{R}}\right), \quad\left(\xi_{+}^{\mathrm{L}}, \xi_{+}^{\mathrm{R}}\right) \mapsto\left(\xi_{+}^{\mathrm{L}}, \xi_{+}^{\mathrm{R}}\right)+\tau(1,1)
$$

and the corrsponding transformations (3.32) and (3.38) for the right wedge $W$ are

$$
\left(\xi_{-}^{\mathrm{L}}, \xi_{+}^{\mathrm{R}}\right) \mapsto\left(e^{2 \pi u} \xi_{-}^{\mathrm{L}}, e^{-2 \pi u} \xi_{+}^{\mathrm{R}}\right), \quad\left(\xi_{-}^{\mathrm{L}}, \xi_{+}^{\mathrm{R}}\right) \mapsto\left(\xi_{-}^{\mathrm{L}}, \xi_{+}^{\mathrm{R}}\right)+\tau(1,1) .
$$

Analogous formulas hold for the backward cone and the left wedge. Hence in the $\xi$ coordinates the transformations have exactly the same form for all $\beta$, including the vacuum case, $\beta=\infty$.

The four coordinate systems $\left(\xi_{ \pm}^{\mathrm{L}}, \xi_{ \pm}^{\mathrm{R}}\right)$ can be put together by defining

$$
\left(\tilde{\xi}^{\mathrm{L}}, \tilde{\xi}^{\mathrm{R}}\right)=(\beta / 2 \pi)\left(\epsilon ( x ^ { \mathrm { L } } ) \left(\exp \left(\epsilon\left(x^{\mathrm{L}}\right) 2 \pi x^{\mathrm{L}} / \beta\right)-1, \epsilon\left(x^{\mathrm{R}}\right)\left(\exp \left(\epsilon\left(x^{\mathrm{R}}\right) 2 \pi x^{\mathrm{R}} / \beta\right)-1\right)\right.\right.
$$

with $\epsilon(x)=1$ for $x \geq 0$ and -1 for $x<0$. This transformation is once continuously differentiable, but second derivatives have a discontinuity on the light cone. The lines corresponding to the flow of the $\Gamma$-groups of the four domains (the forward and backward cones and the two wedges) pass continuously through the boundaries between the domains, although the groups themselves do not merge to a single one parameter unitary group on the Hilbert space.

The transformation (3.56) is of the form $\left(x^{\mathrm{L}}, x^{\mathrm{R}}\right) \mapsto\left(f\left(x^{\mathrm{L}}\right), f\left(x^{\mathrm{R}}\right)\right)$ with $f$ an order preserving bijective map $\mathbf{R} \rightarrow \mathbf{R}$. Hence it is a causal transformation on two-dimensional space-time, i.e., it takes light cones into light cones. Such nonlinear causal maps on Minkowski-space exist only in two space-time dimensions.

Finally we remark that all results of this section hold for general 2D theories, provided the state satisfies a KMS condition with respect to both light cone coordinates, $x^{\mathrm{L}}$ and $x^{\mathrm{R}}$. For factorizing states, this holds automatically as a consequence of the KMS condition with respect to the time direction. A general proof of a KMS condition with respect to light like translations seems out of reach, however, even if one involves the relativistic KMS condition [BB94].

\section{Explicit realizations of modular groups}

In this section we compute explicitly the modular and $\Gamma$-groups for generalized free fields on a light ray and the corresponding tensor product models on $\mathbf{R}^{2}$. In these examples it is possible to discuss the action of the groups on the algebras of double cones and not only of translated forward cones and wedges.

The Weyl algebra of a generalized free Bose field on a light ray is generated by elements $W(f)$, with $f$ a real valued Schwartz test function on $\mathbf{R}$, satisfying the following relations:

$$
W(f)^{*}=W(-f)
$$


and

$$
W(f) W(g)=e^{-K(f, g) / 2} W(f+g)
$$

with

$$
K(f, g)=\int_{-\infty}^{\infty} p Q\left(p^{2}\right) \tilde{f}(-p), \tilde{g}(p) d p
$$

where $Q\left(p^{2}\right)$ is a non-negative polynomial that characterizes the field (see [Y93] ). Here $\tilde{f}(p)=(1 / 2 \pi) \int \exp (-\mathrm{i} p x) f(x) d x$ is the Fourier transform of $f$. The kernel of $\mathcal{K}$ of $K$, defined by $K(f, g)=\int \mathcal{K}(y-x) f(x) g(y) d x d y$, is

$$
\mathcal{K}(y-x)=M(-i d / d y) \delta(y-x)
$$

with $M(p)=p Q\left(p^{2}\right)$, so $W(f)$ and $W(g)$ commute if $f$ and $g$ have disjoint supports.

Translations in time are equivalent to translations along the light ray and are represented by automorphisms of the Weyl algebra,

$$
\alpha_{t}(W(f))=W(f(\cdot-t))
$$

A quasi free KMS state $\omega$ at inverse temperature $\beta$ is defined on the Weyl algebra by

$$
\omega(W(f))=\exp \left(-\omega_{2}(f, f)\right)
$$

where $\omega_{2}$ is given by a positive definite kernel $\mathcal{W}_{2}(y-x)$ (two point function) that is analytic in the strip $S(0, \beta)$ and satisfies

$$
\mathcal{W}_{2}(\xi)-\mathcal{W}_{2}(-\xi)=\mathcal{K}(\xi)
$$

for real $\xi$, together with the KMS condition

$$
\mathcal{W}_{2}(\xi+\mathrm{i} \beta)=\mathcal{W}_{2}(-\xi)
$$

It is straightforward to show that these conditions fix $\mathcal{W}_{2}$ (up to normalization); its Fourier transform is

$$
\tilde{\mathcal{W}}_{2}(p)=\frac{p Q\left(p^{2}\right)}{1-\mathrm{e}^{-\beta p}}
$$

The Fourier transform of the meromorphic function $(1-\exp (-\beta p))^{-1}$ is seen to be $\lim _{\varepsilon \rightarrow 0_{+}}(2 \pi \mathrm{i} \beta)^{-1}\left(\exp \left(\beta^{-1} 2 \pi \xi+\mathrm{i} \varepsilon\right)-1\right)^{-1}$ by contour integration. The Fourier transform of (4.9) for general $Q$ follows by differentiation. In particular we have for $Q \equiv 1$, i.e. a field of scaling dimension 1 ,

$$
\mathcal{W}_{2}(\xi)=\lim _{\varepsilon \rightarrow 0_{+}} \frac{1}{\beta^{2}} \frac{1}{\left(\sinh \frac{\pi(\xi+\mathrm{i} \varepsilon)}{\beta}\right)^{2}},
$$

and for $Q$ a polynomial of degree $n$

$$
\mathcal{W}_{2}(\xi)=\lim _{\varepsilon \rightarrow 0_{+}} \frac{P\left(\cosh \frac{\pi \xi}{\beta}, \sinh \frac{\pi \xi}{\beta}\right)}{\left(\sinh \frac{\pi(\xi+\mathrm{i} \varepsilon)}{\beta}\right)^{2 n+2}}
$$


where $P$ is a polynomial in two variables. We shall restrict ourselves to the case that $Q\left(p^{2}\right)=p^{2 n}$, i.e. a field of a definite scaling dimension $(n+1)$, in order not to mix the effects coming from the non-zero temperature with those due to inhomogeneous polynomials $Q$ (see [Y93] for the latter).

Denoting the Weyl operators corresponding to $Q\left(p^{2}\right)=p^{2 n}$ by $W^{(n)}(f)$ it is clear from (4.3) that we may identify

$$
W^{(n)}(f)=W^{(0)}\left(\mathrm{i}^{n} f^{(n)}\right)
$$

where $f^{(n)}$ is the $n$-the derivative of $f$, and from (4.9) we see also that a KMS state for any $n$ is the same as the KMS state for $n=0$ restricted to the operators $W^{(0)}\left(\mathrm{i}^{n} f^{(n)}\right)$. This will allow us to reduce everything to the simplest case, $n=0$.

Let $\pi$ be the GNS representation defined by the KMS state (4.6) on the Weyl algebra of the $W^{(0)}(f)$ 's. If $I \subset \mathbf{R}$ is an interval, bounded or unbounded, we define $\mathcal{M}^{(n)}(I)$ to be the von Neumann algebra generated by $\pi\left(W^{(n)}(f)\right)$ with supp $f \subset I$. Because of the identification discussed above these algebras are for all $n$ realized on the same Hilbert space.

By exactly the same arguments as in [Y93], Sec. 3, one proves

\subsection{LEMMA:}

If $I$ is an unbounded interval, then $\mathcal{M}^{(n)}(I) \equiv \mathcal{M}(I)$ is independent of $n$. If I is bounded with a non-empty interior, then $\mathcal{M}^{(m)}(I)$ is a proper subalgebra of $\mathcal{M}^{(n)}(I)$ for $m>n$.

This lemma implies in particular that the modular operator $\Delta_{+}$corresponding to the half-line $\mathbf{R}_{+}$is the same for all $n$.

The main result about the modular action is the following:

\subsection{THEOREM:}

Let $\omega$ be the quasi free KMS state (4.6) and $\pi$ the corresponding representation of the Weyl algebra for $n=0$. The modular group of $\mathcal{M}\left(\mathbf{R}_{+}\right)$defined by $\omega$ has the form

$$
\Delta_{+}^{\mathrm{i} u} \pi\left(W^{(0)}(f)\right) \Delta_{+}^{-\mathrm{i} u}=\pi\left(W^{(0)}\left(\delta_{u}^{(0)} f\right)\right)
$$

with

$$
\delta_{u}^{(0)} f(x)=f\left(\frac{\beta}{2 \pi} \log \left\{1+\mathrm{e}^{2 \pi u}\left(\mathrm{e}^{2 \pi x / \beta}-1\right)\right\}\right)
$$

for supp $f \subset \mathbf{R}_{+}$.

Remark 1. It is understood that if supp $f \subset \mathbf{R}_{+}$, then also $\delta_{u}^{(0)} f(x)=0$ for all $x<0$.

Remark 2. The cyclic vector $\Omega$ corresponding to $\omega$ has the Reeh-Schlieder property, in particular it is cyclic for $\mathcal{M}\left(\mathbf{R}_{+}\right)$. Hence (4.13) with supp $f \subset \mathbf{R}_{+}$, together with $\Delta_{+}^{\mathrm{it}} \Omega=$ $\Omega$, already fixes $\Delta_{+}^{\mathrm{i} t}$ as a unitary group on the GNS Hilbert space. But $\Delta_{+}^{\mathrm{i} u} \pi\left(W^{(0)}(f)\right) \Delta_{+}^{-\mathrm{i} u}$ is, of course, a well defined operator on the Hilbert space for all $f$ of compact support, and in fact if $u \geq 0$, then (4.13) and (4.14) hold for functions with support outside of $\mathbf{R}_{+}$with the understanding that $(4.14)$ is zero when the argument of the logarithm is $\leq 0$. This 
is a simple consequence of (3.1)-(3.3). If $u<0$, however, the transformed operator only belongs to the observable algebra if condition (3.3) holds on the support of $f$.

Proof of Theorem 4.2: The formula (4.14) is motivated by Eq. (2.20). In order to show that it is the correct formula for the modular action we have to check the following properties of $\delta_{u}^{(0)}$ :

(i) $\delta_{u}^{(0)}$ maps the space of test functions with support in $\mathbf{R}_{+}$into itself.

(ii) The group property, i.e. $\delta_{u}^{(0)} \circ \delta_{u^{\prime}}^{0}=\delta_{u+u^{\prime}}^{(0)}$.

(iii) The unitarity of $\delta_{u}^{(0)}$ in the scalar product defined by the two point function (4.10).

(iv) The KMS condition: For real test functions $f$ and $g$ with support in $\mathbf{R}_{+}$the function $u \mapsto \omega_{2}\left(f, \delta_{u}^{(0)} g\right)$ has an analytic continuation into the strip $S(-1,0)$ and

$$
\omega_{2}\left(f, \delta_{u-\mathrm{i}}^{(0)} g\right)=\omega_{2}\left(\delta_{u}^{(0)} g, f\right)
$$

Property (i) is obvious from the definition, and (ii) and (iii) are straightforward calculations. We now check the KMS condition. Put

$$
L(u, x):=\frac{\beta}{2 \pi} \log \left\{1+\mathrm{e}^{2 \pi u}\left(\mathrm{e}^{2 \pi x / \beta}-1\right)\right\}
$$

Since $L(u, L(-u, y))=y$ (group property) we have

$$
\omega_{2}\left(f, \delta_{u}^{(0)} g\right)=\iint \mathcal{W}_{2}(L(-u, y)-x) \frac{\partial L(-u, y)}{\partial y} f(x) g(y) d x d y
$$

Using the addition formula for hyperbolic functions, we compute for the two point function (4.10):

$$
\begin{aligned}
& \mathcal{W}_{2}(L(-u, y)-x) \partial L(-u, y) / \partial y \\
&=\frac{1}{4 \beta^{2}}[\sinh (\pi L(-u, y) / \beta) \cosh (\pi x / \beta)-\cosh (\pi L(-u, y) / \beta) \sinh (\pi x / \beta)+\mathrm{i} \varepsilon]^{-2} \frac{\partial L(-u, y)}{\partial y} \\
&=\frac{1}{16 \beta^{2}}\left[\left\{\left(1+\mathrm{e}^{-2 \pi u}\left(\mathrm{e}^{2 \pi y / \beta}-1\right)\right)^{1 / 2}+\left(1+\mathrm{e}^{-2 \pi u}\left(\mathrm{e}^{2 \pi y / \beta}-1\right)\right)^{-1 / 2}\right\} \cosh (\pi x / \beta)\right. \\
&\left.-\left\{\left(1+\mathrm{e}^{-2 \pi u}\left(\mathrm{e}^{2 \pi y / \beta}-1\right)\right)^{1 / 2}+\left(1+\mathrm{e}^{-2 \pi u}\left(\mathrm{e}^{2 \pi y / \beta}-1\right)\right)^{-1 / 2}\right\} \sinh (\pi x / \beta)+\mathrm{i} \varepsilon\right]^{-2} \times \\
& \times \frac{\mathrm{e}^{-2 \pi u} \mathrm{e}^{2 \pi y / \beta}}{\mathrm{e}^{-2 \pi u}\left(\mathrm{e}^{2 \pi y / \beta}-1\right)+1} \\
& \frac{1}{16 \beta^{2}}\left[\left\{\mathrm{e}^{-2 \pi u}\left(\mathrm{e}^{2 \pi y / \beta}-1\right)\right\} \cosh (\pi x / \beta)-\left\{2+\mathrm{e}^{-2 \pi u}\left(\mathrm{e}^{2 \pi y / \beta}-1\right)\right\} \sinh (\pi x / \beta)+\mathrm{i} \varepsilon\right]^{-2} \times \\
& \times \mathrm{e}^{-2 \pi u} \mathrm{e}^{2 \pi y / \beta} \\
&=\frac{\mathrm{e}^{2 \pi y / \beta}}{16 \beta^{2}}\left[\mathrm{e}^{-\pi u}\left(\mathrm{e}^{2 \pi y / \beta}-1\right)[\cosh (\pi x / \beta)-\sinh (\pi x / \beta)]-\mathrm{e}^{\pi u} 2 \sinh (\pi x / \beta)+\mathrm{i} \varepsilon\right]^{-2} .
\end{aligned}
$$


For $x, y>0, e^{-\pi u}$ comes with a positive factor and $e^{\pi u}$ with a negative one. For $\varepsilon>0$, the total expression is therefore analytic in $u$ in the strip $S(-1,0)$, and this analyticity is preserved in the limit $\varepsilon \rightarrow 0_{+}$after smearing in $x$ and $y$ with test functions with support in $\mathbf{R}_{+}$. The boundary value at $u-i, u \in \mathbf{R}$, is

$$
\frac{\mathrm{e}^{2 \pi y / \beta}}{16 \beta^{2}}\left[\mathrm{e}^{\pi u} 2 \sinh (\pi x / \beta)-\mathrm{e}^{-\pi u}\left(\mathrm{e}^{2 \pi y / \beta}-1\right)[\cosh (\pi x / \beta)-\sinh (\pi x / \beta)]+\mathrm{i} \varepsilon\right]^{-2} .
$$

This is precisely $\mathcal{W}_{2}(x-L(-u, y)) \partial L(-u, y) / \partial y$ (by the same computation). Hence the KMS condition is verified.

The representation of the group $\Gamma_{+}(\tau)=\exp \left(\mathrm{i} \tau G_{+} / \beta\right)$ with the positive generator $G_{+} / \beta=H+(1 / \beta) \log \Delta_{+}$now follows immediately from Eqs (2.25) and (4.13)-(4.14):

\subsection{THEOREM:}

For $\tau \geq 0$ and all $f$

$$
\Gamma_{+}(\tau) \pi\left(W^{(0)}(f)\right) \Gamma_{+}(-\tau)=\pi\left(W^{(0)}\left(\gamma_{\tau}^{(0)} f\right)\right)
$$

with

$$
\gamma_{\tau}^{(0)} f(x)=f\left(x+\frac{\beta}{2 \pi} \log \left\{1-\frac{2 \pi \tau}{\beta} \mathrm{e}^{-2 \pi x / \beta}\right\}\right)
$$

Remark: It is understood that $\gamma_{\tau}^{(0)} f(x)=0$ if the argument of the logarithm is $\leq 0$, i.e., if $x \leq-\beta /(2 \pi) \log (2 \pi \tau / \beta)$. Note that if $\tau \geq \beta /(2 \pi)$, then supp $\gamma_{\tau}^{(0)} f \subset \mathbf{R}_{+}$for any $f$ of compact support.

By (4.12) we obtain as a corollary of Theorems 4.2 and 4.3

\subsection{THEOREM:}

For $n>0$ the action of ad $\Delta_{+}^{\mathrm{i} u}$ and $\operatorname{ad} \Gamma_{+}(\tau)$ on $W^{(n)}(f)$ with supp $f \subset \mathbf{R}_{+}$is

$$
\Delta_{+}^{\mathrm{i} u} \pi\left(W^{(n)}(f)\right) \Delta_{+}^{-\mathrm{i} u}=\pi\left(W^{(n)}\left(\delta_{u}^{(n)} f\right)\right)
$$

with

$$
\delta_{u}^{(n)} f(x)=\int_{0}^{x} d x_{1} \int_{0}^{x_{1}} \cdots \int_{0}^{x_{n-1}} d x_{n} \delta_{u}^{(0)} f^{(n)}\left(x_{n}\right)
$$

and for $\tau \geq 0$

$$
\Gamma_{+}(\tau) \pi\left(W^{(n)}(f)\right) \Gamma_{+}(-\tau)=\pi\left(W^{(n)}\left(\gamma_{\tau}^{(n)} f\right)\right)
$$

with

$$
\gamma_{\tau}^{(n)} f(x)=\int_{0}^{x} d x_{1} \int_{0}^{x_{1}} \cdots \int_{0}^{x_{n-1}} d x_{n} \gamma_{\tau}^{(0)} f^{(n)}\left(x_{n}\right)
$$

Remark. It should be noted that $\delta_{u}^{(n)} f$ is in general no longer a test function if $n>0$, for it may behave like $x^{n-1}$ for $x \rightarrow \infty$. However, it belongs to the Hilbert space defined 
by the two point function and hence the Weyl operators are well defined. The same applies to $\gamma_{\tau}^{(n)} f$.

Next we investigate the localization properties of the modular groups. We recall from Lemma 4.1 that for an unbounded interval $\left[x, \infty\left[\right.\right.$ the algebras $\mathcal{M}^{(n)}([x, \infty[) \equiv \mathcal{M}([x, \infty[)$ are independent of $n$. Hence the general result (3.1) applies. For the algebras corresponding to bounded intervals we have

\subsection{THEOREM:}

For $-\infty<x<y<\infty$ and $u$ and $\tau$ restricted according to (3.3), (3.5) (3.9), (3.12)

$$
\operatorname{ad} \Delta_{+}^{\mathrm{i} u} \mathcal{M}^{(0)}([x, y])=\mathcal{M}^{(0)}\left(\left[\varphi_{+}(u, x), \varphi_{+}(u, y)\right]\right)
$$

and

$$
\operatorname{ad} \Gamma_{+}(\tau) \mathcal{M}^{(0)}([x, y])=\mathcal{M}^{(0)}\left(\left[\psi_{+}(\tau, x), \psi_{+}(\tau, y)\right]\right) .
$$

For $n>0$ a local algebra $\left.\mathcal{M}^{(n)}([x, y])\right)$ is not mapped into an $\mathcal{M}^{(n)}(I)$ with bounded $I$.

Proof: For fixed $u$ and $\tau$ the maps $x \mapsto \varphi_{+}(u, x)$ and $x \mapsto \psi_{+}(\tau, x)$ are one to one for $x$ satisfying (3.3) and (3.9) respectively, and the inverse maps correspond to $u \rightarrow-u$ and $\tau \rightarrow-\tau$. From (3.14) it is clear that $f$ has its support in $[x, y]$, iff $\delta_{u}^{(0)} f$ has its support in $\left[\varphi_{+}(u, x), \varphi_{+}(u, y)\right]$ iff $\gamma_{\tau}^{(0)} f$ has its support in $\left[\psi_{+}(\tau, x), \psi_{+}(\tau, y)\right]$. Hence (4.23) and (4.24) follows directly from Theorems 4.2 and 4.3 .

To show the dislocalization for $n>0$ we note first that neither $\delta_{u}^{(0)} f^{(n)}$ nor $\gamma_{\tau}^{(0)} f^{(n)}$ is a derivative of a function with compact support (except for $f \equiv 0$ ). This is easily seen by considering the Fourier transforms of these functions, divided by $p$; the $1 / p$ singularity is not compensated by the derivatives because of the non-linear variable transformations, and analyticity is lost. Consider now a bounded interval $I$ and a function $g$ such that $g^{(n+1)}$ vanishes on $I$. Then $W^{(0)}(g)$ belongs to the commutant of $\mathcal{M}^{(n)}(I)$. If $W^{(n)}\left(\delta_{u}^{(n)} f\right)=$ $W^{(0)}\left(\delta_{u}^{(0)} f^{(n)}\right)$ would belong to $\mathcal{M}^{(n)}(I)$, then it would commute with $W^{(0)}(g)$, which means that

$$
\int \delta_{u}^{(0)} f^{(n)}(x) g^{\prime}(x) d x=0 .
$$

This must in particular hold for all $g$ with $g^{\prime} \equiv 1$ on $I$ because such $g$ fulfill $g^{(n+1)}=0$ on $I$ for $n>0$. Hence

$$
\int_{I} \delta_{u}^{(0)} f^{(n)}(x) d x=0 .
$$

By isotony this should also hold for all larger intervals, and hence $\delta_{u}^{(0)} f^{(n)}$ would be a derivative of a function of compact support. As remarked above, this is not the case, and we have a contradiction to the assumption that $W^{(n)}\left(\delta_{u}^{(n)} f\right)$ belongs to $\mathcal{M}^{(n)}(I)$ with $I$ bounded. By the same argument $W^{(n)}\left(\gamma_{\tau}^{(n)} f\right)$ does not belong to $\mathcal{M}^{(n)}(I)$.

Remark 1. In terms of the field operators $\Phi^{(n)}(x)$, defined by

$$
\pi\left(W^{(n)}(f)\right)=\exp \left(\mathrm{i} \int \Phi^{(n)}(x) f(x) d x\right),
$$


Eqs. (3.13) and (3.17) say that

$$
\Delta_{+}^{\mathrm{i} u} \Phi^{(0)}(x) \Delta_{+}^{-\mathrm{i} u}=\Phi^{(0)}\left(\varphi_{+}(u, x)\right) \frac{\partial \varphi_{+}(u, x)}{\partial x}
$$

and

$$
\Gamma_{+}(\tau) \Phi^{(0)}(x) \Gamma_{+}(-\tau)=\Phi^{(0)}\left(\psi_{+}(\tau, x)\right) \frac{\partial \psi_{+}(\tau, x)}{\partial x} .
$$

In particular we have

$$
\Delta_{+}^{\mathrm{i} u} \Phi^{(0)}(0) \Delta_{+}^{-\mathrm{i} u}=e^{-2 \pi u} \Phi^{(0)}(0)
$$

and

$$
\Gamma_{+}(\tau) \Phi^{(0)}(0) \Gamma_{+}(-\tau)=(1+(2 \pi \tau / \beta))^{-1} \Phi^{(0)}((\beta / 2 \pi) \log (1+(2 \pi \tau / \beta)))
$$

(Although the field is only an operator valued distribution, these equations have a rigorous meaning in terms of quadratic forms.) Conversely (3.33) and (3.34), together with Eqs. (2.20) and (2.26), imply (3.31) and (3.32). For $n>0$, however, $\operatorname{ad} \Delta_{+}^{\mathrm{i} u}$ is a non-local transformation of the field operators by Thm. 4.4. For instance we have

$$
\Delta_{+}^{\mathrm{i} u} \Phi^{(1)}(0) \Delta_{+}^{-\mathrm{i} u}=e^{-2 \pi u} \Phi^{(1)}(0)-(2 \pi / \beta) e^{-4 \pi u} \int_{0}^{\infty} \Phi^{(1)}(x) d x .
$$

This shows clearly that there is more to the transformation law for the fields than Eqs. (2.20) and (2.29) alone.

If $\mathcal{M}\left(\mathbf{R}_{+}\right)$is replaced by $\mathcal{M}\left(\mathbf{R}_{-}\right)$the previous results apply with appropriate changes of signs, cf. (3.5).

Forming tensor product algebras as in (3.13) we obtain generalized free fields on two dimensional space-time and KMS states that factorize in the light cone variables. In the case of the field with lowest scaling dimension, i.e., $n=0$, the double cone algebras $\mathcal{M}^{(0)}\left(I_{\mathrm{L}} \times I_{\mathrm{R}}\right)$, with $I_{\mathrm{L}}, I_{\mathrm{R}}$ bounded intervals, are again mapped into algebras of double cones. The flow lines of Figs. 1-4 describe in this case not only the movement of the apex of a forward light cone or the edge of a wedge, but also the movement of the double cones.

For fields of higher scaling dimension, i.e. $n>0$, however, double cone algebras are after the transformation no longer localized in double cones within the net $\mathcal{M}^{(n)}$. They are still localized in double cones within the net $\mathcal{M}^{(0)}(\cdot)$, because $\mathcal{M}^{(n)}(\cdot)$ is a subnet of $\mathcal{M}^{(0)}(\cdot)$.

\section{Conclusions}

In a KMS state at inverse temperature $\beta$ the time translations coincide (up to a sign and scaling by $\beta$ ) with the modular group of the global observable algebra. From this fact, 
and the general theory of half-sided modular inclusions, algebraic relations between time translations and the modular groups for certain domains of space-time can be derived. The action of the modular groups on observables localized inside these domains far from the boundary is approximately given by the time translations. In two dimensional models and states that satisfy a KMS condition with respect to light-like translations (in particular models that factorize in the light cone coordinates), a geometric interpretation can be given of the action of the modular groups of the forward light cone and a space-like wedge on observable algebras localized in translated domains of the same type. This action can be studied in detail in simple free field models. Besides the modular groups, the theory also leads to one parameter groups with positive generators, for which the KMS state is a ground state. The actions of these groups for the forward cone and the wedge can also be described geometrically and interpreted, at least formally, as a kind of a reverse Unruh effect.

\section{Acknowledgements}

We thank D. Buchholz, H. Narnhofer, P. Michor and W. Thirring for helpful comments. Hospitality of the the Erwin Schrödinger Institute, Vienna (H.J.B.) and the University Science Institute, Reykjavik (J.Y.) is also gratefully acknowledged.

\section{References}

[BB94] J. Bros, D. Buchholz: Towards a relativistic KMS-condition, Nucl. Phys. B 429,2911-318 (1994).

[BW75] J. Bisognano and E.H. Wichmann: On the duality condition for a Hermitean scalar field, J. Math. Phys. 16, 985-1007 (1975).

[BW76] J. Bisognano and E.H. Wichmann: On the duality condition for quantum fields, J. Math. Phys. 17, 303-321 (1976).

[Bch92] H.-J. Borchers: The CPT-Theorem in Two-dimensional Theories of Local Observables Commun. Math. Phys. 143, 315-332 (1992).

[Bch95] H.-J. Borchers: On the use of modular groups in quantum field theory, Ann. Inst. H. Poincaré 64, 331-382 (1996).

[Bch98] H.-J. Borchers: Half-sided Translations and the Type of von Neumann Algebras, Lett.Math.Phys., to appear (1998)

[BR79] O. Bratteli, D.W. Robinson: Operator Algebras and Quantum Statistical Mechanics I, Springer Verlag, New York, Heidelberg, Berlin (1979).

[Bu78] D. Buchholz: On the Structure of Local Quantum Fields with Non-trivial Interactions, In: Proceedings of the International Conference on Operator Algebras, Ideals and their Applications in Theoretical Physics, Leipzig 1977, Teubner-Texte zur Mathematik (1978) p. 146-153.

[BDL90] D. Buchholz, C. D’Antoni, R. Longo: Nuclear Maps and Modular Structures II: Applications to Quantum Field Theory, Commun. Math. Phys. 129, 115-138 (1990). 
[ENTS95] G.G. Emch, H. Narnhofer, W. Thirring, G.L. Sewell: Anosov actions on noncommutative algebras, J. Math. Phys. 35, 5582-5598 (1994)

[Ha96] R. Haag: Local Quantum Physics, Springer Verlag, 2nd ed., Berlin-Heidelberg-New York (1996).

[HL82] P.D. Hislop and R. Longo: Modular structure of the local algebra associated with a free massless scalar field theory, Commun. Math. Phys. 84, 71-85 (1982).

[KR86] R.V. Kadison and J.R. Ringrose: Fundamentals of the Theory of Operator Algebras II, New York: Academic press, (1986).

[RS61] H. Reeh and S. Schlieder: Eine Bemerkung zur Unitäräquivalenz von Lorentzinvarianten Feldern, Nuovo Cimento 22, 1051 (1961).

[Sew80] G.L. Sewell: Relativity of temperature and the Hawking effect, Phys. Lett. 79A, 23-24 (1980)

[Sew82] G.L. Sewell: Quantum fields on manifolds: PCT and gravitationally induced thermal states, Ann. Phys. 141, 201-224 (1982)

[Ta70] M. Takesaki: Tomita's Theory of Modular Hilbert Algebras and its Applications, Lecture Notes in Mathematics, Vol. 128 Springer-Verlag Berlin, Heidelberg, New York (1970).

[U76] W.G. Unruh: Notes on black-hole evaporation, Phys. Rev. D14, 870-892 (1976)

[Wie92] H.-W. Wiesbrock: A comment on a recent work of Borchers, Lett.Math.Phys. 25, 157-159 (1992).

[Wie93] H.-W. Wiesbrock: Half-Sided Modular Inclusions of von Neumann Algebras, Commun. Math. Phys. 157, 83 (1993)

[Wie97] H.-W. Wiesbrock: Half-Sided Modular Inclusions of von Neumann Algebras, Erratum, Commun. Math. Phys. 184, 683-685 (1997)

[Y93] J. Yngvason: A note on Essential Duality, Lett. Math. Phys. 31,127-141 (1993) 


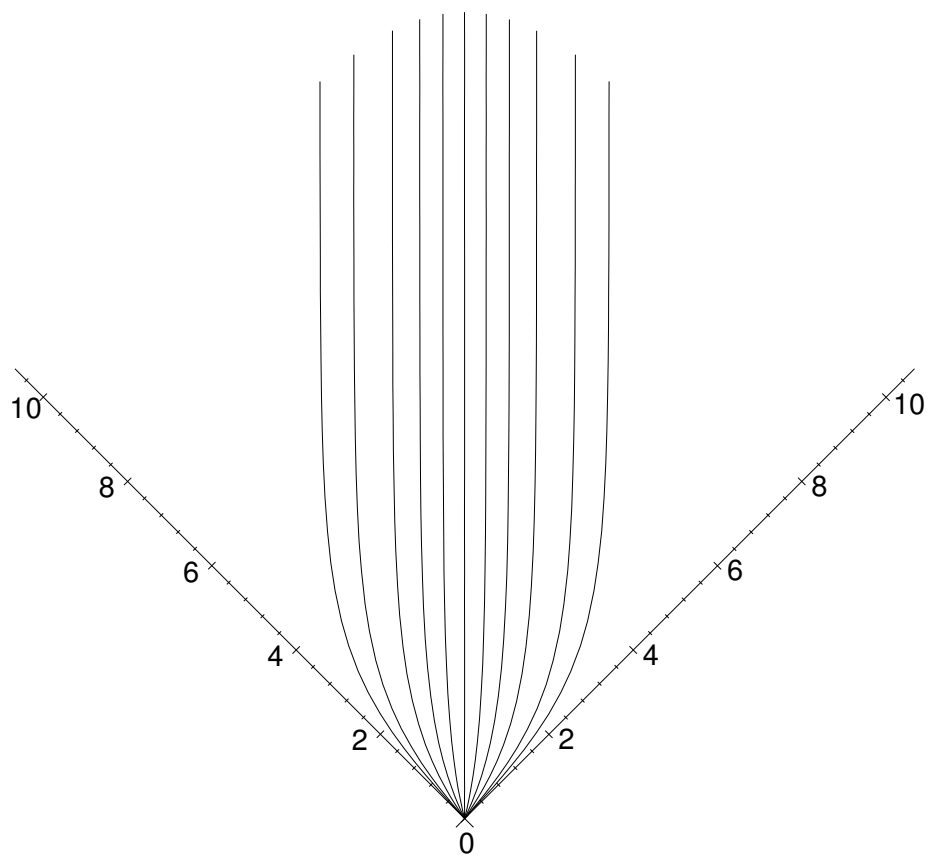

Figure 1: The modular flow in the forward light cone. The unit is inverse temperature, $\beta$.

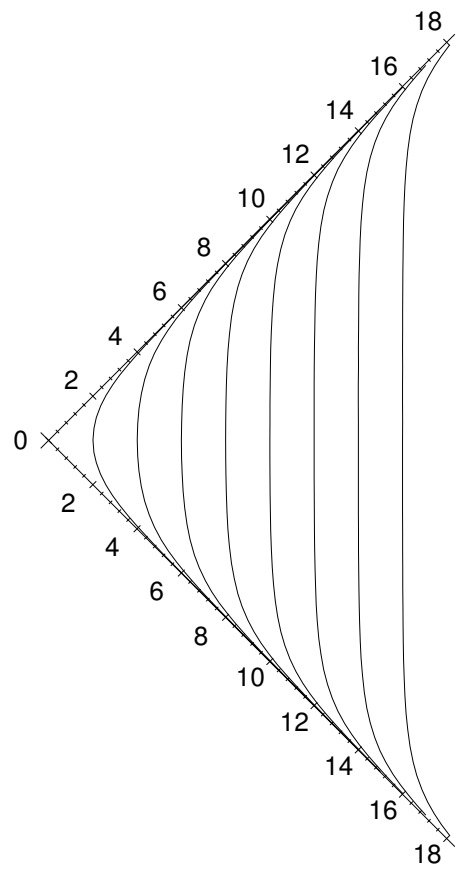

Figure 2: The modular flow in a space-like wedge 


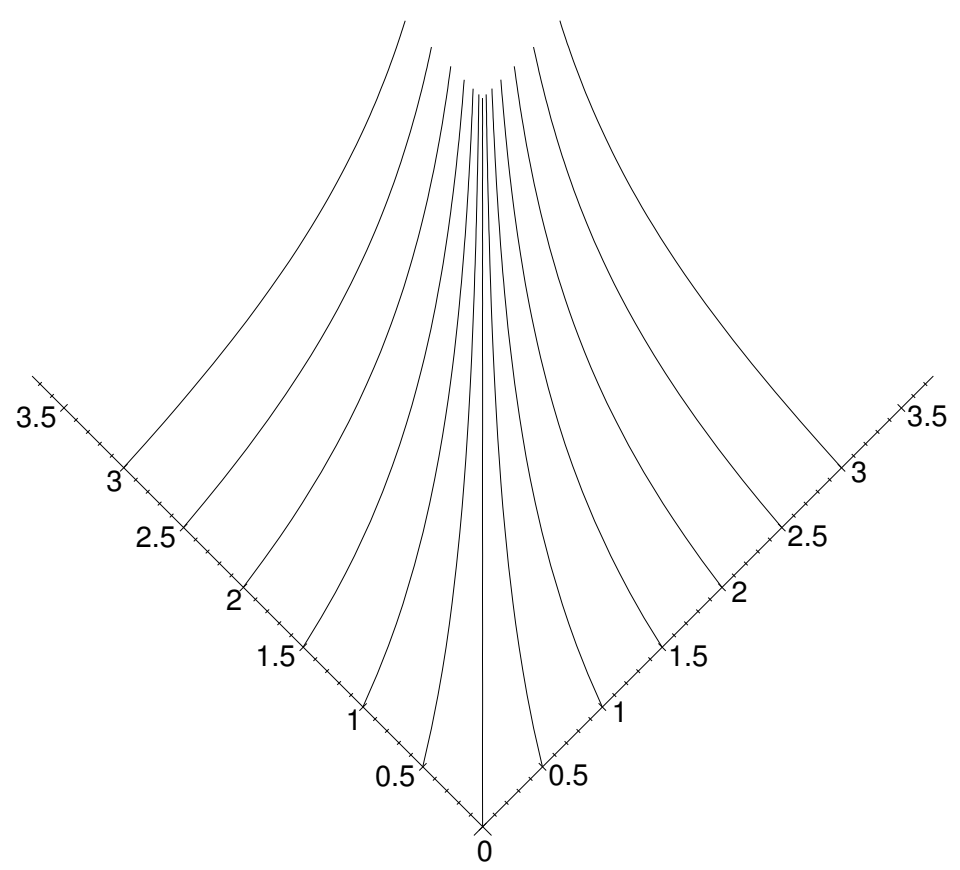

Figure 3: The flow of $\Gamma_{\mathrm{V}^{+}}(\tau)$ within the forward light cone. The whole pattern is invariant under translations in the $x^{0}$-direction.

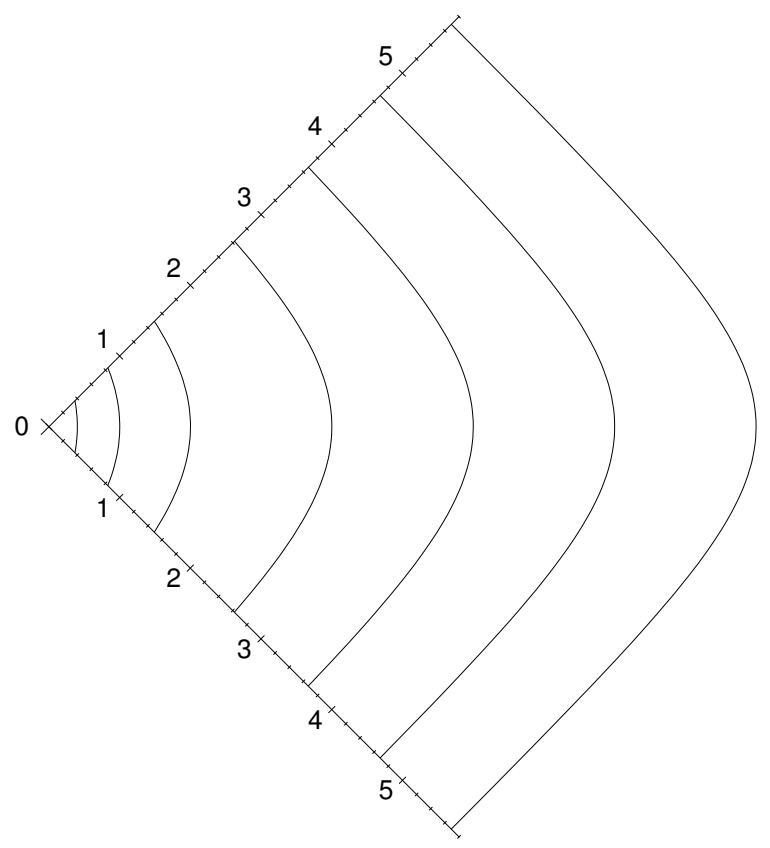

Figure 4: The flow of $\Gamma_{W}(\tau)$ in a space like wedge. The whole pattern is invariant under translations in the $x^{1}$-direction. 